\title{
OBSTRUCTION THEORY AND MULTIPARAMETER HOPF BIFURCATION ${ }^{1}$
}

BY

JORGE IZE

\begin{abstract}
The Hopf bifurcation problem is treated as an example of an equivariant bifurcation. The existence of a local bifurcating solution is given by the nonvanishing of an obstruction to extending a map defined on a complex projective space and is computed using the complex Bott periodicity theorem. In the case of the classical Hopf bifurcation the results of Chow, Mallet-Paret and Yorke are recovered without using any special index as the Fuller degree: There is bifurcation if the number of exchanges of stability is nonzero. A global theorem asserts that the sum of the local invariants on a bounded component of solutions must be zero.
\end{abstract}

I. Introduction. Several topological methods have been used in the study of the Hopf bifurcation problem (see [AY, I1, I2, CMY]). The basic idea of this paper is to consider the existence of a periodic solution to an autonomous differential equation as an obstruction to the construction of an extension for equivariant mappings, in a sense which will be clear later. It should be pointed out that this obstruction approach is not limited to problems with an $S^{1}$ action and that it should work in different contexts.

In the Hopf bifurcation problem one looks for periodic solutions of the equation

$$
d X / d t=L(\mu) X+g(\mu, X) \equiv f(\mu, X),
$$

where $X \in \mathbf{R}^{M}, \mu \in \mathbf{R}^{k-1}, k \geqslant 2, L(\mu)$ is a $C^{0.1}$ family of $M \times M$ matrices and $g$ is $C^{1,1}$ in $X$ and $C^{0,1}$ in $\mu$. Furthermore the following hypotheses hold:

H.1. $g(\mu, 0)=0, g(\mu, X)=o(X)$ as $X$ tends to $0, L(0)$ is invertible.

H.1 implies that $X=0$ is a stationary solution and that there is no small norm stationary solution for $\mu$ close to 0 , but $X=0$.

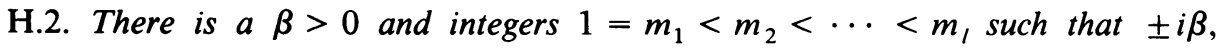
$\pm \operatorname{im}_{2} \beta, \ldots, \pm \mathrm{im}_{1} \beta$ belong to the spectrum of $L(0)$ and no other eigenvalue of $L(0)$ has the form $m \beta, m$ an integer.

H.2 implies that $\dot{X}=L(0) X$ has periodic solutions with periods $2 \pi / \beta$, $2 \pi / m_{2} \beta, \ldots, 2 \pi / m_{1} \beta$. It is easy to see that, if $L(0)$ has no purely imaginary

Received by the editors November 23, 1982 and, in revised form, August 17, 1984.

1980 Mathematics Subject Classification. Primary 58F14; Secondary 55S35.

Key words and phrases. Hopf bifurcation, equivariant obstruction.

${ }^{1}$ This research was done while the author was visiting the IMSP, Universite de Nice, the Centre d'Orsay, Université de Paris-Sud and the Forschungsschwerpunkt Dynamische Systeme, Universität Bremen. 
eigenvalues, then the full equation cannot have periodic solutions close to $X=0$, $\mu=0$. On the other hand, $L(0)$ may have other purely imaginary eigenvalues since there is no other condition on its spectrum.

H.3. Let $d_{j}$ be the algebraic multiplicity of $\operatorname{im}_{j} \beta$ and let $\alpha_{j, k_{0}}(\mu)+i\left(m_{j} \beta+\gamma_{j, k_{0}}(\mu)\right)$ with $\alpha_{j, k_{0}}(0)=\gamma_{j, k_{0}}(0)=0, k_{0}=1, \ldots, d_{j}, j=1, \ldots, l$, be the corresponding eigenvalues of $L(\mu)$, counted with multiplicity $\left(\alpha_{j, k_{0}}(\mu)\right)$ and $\gamma_{j, k_{0}}(\mu)$ are continuous in $\mu$ : see [12]). Assume that $\alpha_{j, k_{0}}(\mu) \neq 0$ for $\mu$ small, $\mu \neq 0$.

H.3 is a transversality condition and implies that $\dot{X}=L(\mu) X$ has no periodic solution with period close to $2 \pi / \beta, \ldots, 2 \pi / m_{l} \beta$, for $\mu \neq 0$.

For the case of a one-parameter problem $(k=2)$, let $n_{j}^{ \pm}$be the number of eigenvalues crossing from left to right (respectively from right to left) the imaginary axis at $\operatorname{im}_{j} \beta$ as $\mu$ passes through 0 . More precisely, $n_{j}^{+}$is the number of $\alpha_{j, k}(\mu)$ which pass from negative values to positive values. Set $n_{j}=n_{j}^{+}-n_{j}^{-}$. It is easy to see that $n_{j}$ is independent of the choice in numbering the eigenvalues.

The first result for global Hopf bifurcation was given by Alexander and Yorke in 1974 , using cohomology theory.

THEOREM 1. If $k=2$ and $\left|\sum_{j=1}^{l} n_{j}\right|$ is odd, then there is a continuum in $\mathbf{R}^{M} \times \mathbf{R} \times \mathbf{R}^{+}$ of points $\left(X_{0}, \mu, T\right)$, corresponding to nontrivial periodic solutions, starting at $X_{0}$ and of period (not necessarily minimal) $T$. This continuum branches off $(0,0,2 \pi / \beta)$ and is either unbounded or goes to another point $\left(X_{1}, \mu_{1}, T_{1}\right)$ with $L\left(\mu_{1}\right) X_{1}+g\left(\mu_{1}, X_{1}\right)=0$ a stationary solution.

This theorem was later proved in [I1], using homotopy arguments, as a special example of a two-parameter bifurcation problem, and extended to abstract parabolic equations in [I2].

In fact it is easy to see that it is enough to have one of the $n_{j}$ 's odd to get the same conclusion, with the continuum starting at $\left(0,0,2 \pi / m_{j} \beta\right)$, since there is no conclusion on the minimality of the period.

Theorem 1 was then improved by Chow, Mallet-Paret and Yorke in 1978 to the following:

THEOREM 2. If $k=2$ and $\sum_{j=1}^{l} n_{j} / m_{j} \neq 0$, then the same conclusion holds. In fact it is enough to have one of the $n_{j}$ 's $\neq 0$.

The proof of this theorem relies on a degree, introduced by Fuller, for periodic orbits for ordinary differential equations. The change of degree for $\mu$ passing through 0 is exactly the above sum.

Although the Fuller's degree has all the properties of the usual degree, its nonvanishing does not guarantee the minimality of the period (see the examples in [I1] or in [AY]) and is not necessary for bifurcation in the sense that if the degree is zero one should be able to find a nonlinearity without periodic solutions. This last statement is of course not a rigorous one but rather a feeling which will be made more precise, using the obstruction approach for multiparameter bifurcation, in a subsequent paper. From Theorem 2 it seems that a "necessary" condition would be that some $n_{j}$ is nonzero. 
The present paper is then an attempt to understand the Fuller's degree, in particular the specificity of autonomous differential equations (giving equivariant bifurcation equations), and to try to answer the above question.

In this context, the following result, together with some extensions and corollaries given in the text, will be proved:

MAIN THEOREM. (1) If $2 \leqslant k \leqslant 2 M$, there are integers $n_{j}(k), j=1, \ldots, l$, such that if $n_{j}(k) \neq 0$ then there is a continuum in $C^{0}\left(\mathbf{R}^{M}\right) \times \mathbf{R}^{k} \times \mathbf{R}^{+}$of periodic solutions of period $T$ (not necessarily minimal) branching off $\left(0,0,2 \pi / m_{j} \beta\right)$ and either being unbounded or going to another stationary point. $n_{j}(k)$ is the class of im ${ }_{j} \nu I-L(\mu)$, for $\|\mu\|+|\nu-\beta|=\rho$ small, in the stable group $\Pi_{k-1}\left(\mathrm{GL}\left(\mathbf{C}^{M}\right)\right.$ ) (which is, according to Bott's periodicity theorem, $Z$ if $k$ is even, 0 if $k$ is odd ) and $n_{j}(2)$ is the above $n_{j}$.

(2) If the continuum is bounded and each stationary point on it, $\left(X_{p}, \mu_{p}, T_{p}\right), p$ in a finite set $I$, is such that the linearization of $f(\mu, X)$ at $\left(\mu_{p}, X_{p}\right)$ has the form of $(*)$ with $L(\mu)$ replaced by $L_{p}(\lambda)$ and satisfies H.1, H.2, H.3, then the following formula holds:

$$
0=\sum_{p \in I} \operatorname{Sign} \operatorname{det} L_{p}\left(\mu_{p}\right) \sum_{j=1, \ldots, l_{p}} n_{j}(k, p) /\left(m_{j}(p)\right)^{k / 2},
$$

where $n_{j}(k, p), m_{j}(p)$ are the corresponding integers for $\left(X_{p}, \mu_{p}\right)$ and all eigenvalues of $L_{p}(\mu)$ of the form $m_{j}(p) 2 \pi / T_{p}$.

Note that for $k=2$ one recovers the Fuller's degree result. The unstable case $(k>2 M)$ will also be studied and it will be seen that if all $n_{j}(k)$ are zero, then there is a nonlinear part so that the bifurcation equations have no nontrivial solution (see §II.4).

Despite its length, this paper is based on a rather simple topological idea: in the local bifurcation problem, one looks for a solution of $(*)$ with $\|X\|=\varepsilon$ for any small $\varepsilon$. From the hypotheses, it is easy to see that there is no such solution if $\|\mu\|+|\nu-\beta|$ $=\rho$ and $\varepsilon$ is small enough. One then changes the question of finding a zero to (*) to proving that the equation (*), restricted to the set $\{(\mu, \nu, X) /\|\mu\|+|\nu-\beta|=\rho$, $\|X\|=\varepsilon\}$, has no nonzero equivariant extension to the set $\{(\mu, \nu, X) /\|\mu\|+|\nu-\beta|$ $\leqslant \rho,\|X\|=\varepsilon\}$. In particular, (*) itself must have a zero, with $\|X\|=\varepsilon$. This new setting has the advantage of allowing deformations of $(*)$ and hence to give sufficient conditions for existence of solutions. Equivariant obstruction theory is then the topological machinery which formalizes this idea. The main characteristics of this tool are recalled in §II.3 and a step-by-step construction of the extension is given in Remark II.4.b.

It is then clear that the same idea is applicable to any bifurcation problem which is equivariant under the action of some compact Lie group. The only difficulty is to compute the obstructions (this is the subject of a paper in preparation). In the present case, the Lie group is $S^{1}$, corresponding to translation along orbits. If one works in the space of orbits (taking the quotient by the action or fixing the phase in terms of classical Hopf bifurcation), one is lead to consider complex projective spaces. On the other hand, it should be pointed out that the same technique works, without any change, to any bifurcation problem with an $S^{1}$ action: instead of a 
Fourier decomposition, one has a decomposition on irreducible subspaces. For example [Mt] considers a problem with a free action of $S^{1}$ on the space variable $X{ }^{2}$ Other concrete examples may be found in [AY, CMY, FR, I1, 12], and in Remark II.4.5.

It is important to observe that the $k$ parameters in this problem have a crucial role. However, in many concrete models, one has additional dummy parameters. One may then apply the results of this paper to each fixed value of these new parameters and expect a "surface" of bifurcating solutions. This "surface" should have the dimension of the space of dummy parameters plus one (coming from the dimension of the orbits). That this is so is proved in [IMPV] and, for the general equivariant case, in a paper in preparation with the same authors.

A final comment about the organization of the paper is in order: One of the main purposes of this research is to apply the method of obstruction theory to a problem in analysis. This implies that the proof of the Main Theorem spans the whole paper and that the flow of the reasoning is interrupted by a fair number of remarks. These are either of an explanatory character, or explore alternate routes, or go deeper into the topology. In most cases the point where the main proof resumes is marked so that, in a first lecture, there is no break in the reading process. Some of the basic tools of nonlinear functional analysis are assumed without precise reference. They may be found in any standard text on the subject, for instance in [Ni]. Throughout the paper, the degree of a map stands for the Brouwer degree at 0 for a map defined on a ball, and is denoted by deg. The class of a map is the homotopy class, the homotopy group is clear according to the context. The stable class of a matrix, the integers $n_{j}(k)$, are denoted Degree.

In the next section the local bifurcation result will be proved and in the following part the global bifurcation will be considered.

\section{Local bifurcation.}

II.1. The bifurcation equations.

Contrary to the preceding articles, where the geometrical space $\mathbf{R}^{M}$ was considered, the bifurcation problem will be studied in the space of $2 \pi$-periodic functions, where the group action is explicitly given in terms of Fourier series.

To begin, the problem is normalized by fixing the period to be $2 \pi$. Thus put $\tau=\nu t$ and $(*)$ becomes

$$
\nu \dot{X}=L(\mu) X+g(\mu, X),
$$

where $\dot{X}=d X / d \tau .2 \pi$-periodic solutions of $(*)_{\nu}$ correspond to $2 \pi / \nu$-periodic solutions of $(*)$.

Secondly, the reals mod $2 \pi$ are identified to $S^{1}$. The spaces $W^{1,2}\left(S^{1}\right), L^{2}\left(S^{1}\right)$ of real-valued functions $x: S^{1} \rightarrow \mathbf{R}$, with inner products

$$
\left(x_{1}, x_{2}\right)_{\varepsilon}=\frac{1}{2 \pi} \int_{0}^{2 \pi}\left(x_{1} x_{2}+\varepsilon \dot{x}_{1} \dot{x}_{2}\right) d \tau, \quad \varepsilon=1,0,
$$

\footnotetext{
${ }^{2}$ The author wishes to thank the referee for indicating this reference to him.
} 
are identified with the space of Fourier series $\sum_{-\infty}^{\infty} x_{n} e^{i n \tau}$, with $x_{-n}=\bar{x}_{n}$ and $\sum_{-\infty}^{\infty}\left(1+\varepsilon n^{2}\right)\left|x_{n}\right|^{2}<\infty$. Then let $E$ be $L^{2}\left(S^{1}\right)^{M}$ and let $B$ be $W^{1,2}\left(S^{1}\right)^{M}$; it is straightforward to check that $(*)_{\nu}$ defines a $C^{1}$ map from $\mathbf{R}^{k} \times B$ into $E$ and that $\nu \dot{X}-L(\mu) X$ is a Fredholm operator of index 0 from $B$ to $E$. Thus $(*)_{\nu}$ is equivalent (by projection on the Fourier series basis for $E$ ) to the infinite-dimensional system

$$
(\text { ivnI }-L(\mu)) X_{n}-g_{n}\left(\mu, X_{0}, X_{1}, \ldots\right)=0 \text { for } n \geqslant 0 .
$$

Here $X(\tau)=\sum_{-\infty}^{\infty} X_{n} e^{i n \tau}, X_{n} \in \mathbf{C}^{M}, X_{-n}=\bar{X}_{n}$, is an element of $B$ and $g_{n}\left(\mu, X_{0}, X_{1}, \ldots\right)=(1 / 2 \pi) \int_{0}^{2 \pi} g(\mu, X(\tau)) e^{-i n \tau} d \tau$.

Since $\nu$ is close to $\beta$ and $\mu$ close to 0 it is clear that, from hypotheses H.1-H.3, the linear part $i \nu n I-L(\mu)$ will be singular only for $\nu=\beta, \mu=0$ and $n=m_{1}, \ldots, m_{l}$. Hence one can reduce locally the system to a finite-dimensional bifurcation equation with the following argument: Write $X(\tau)$ as

$$
X(\tau)=\sum_{j=-l}^{l} X_{j} e^{i m_{j} \tau}+Y(\tau)
$$

where $m_{-j} \equiv-m_{j}, X_{-j}=\bar{X}_{j}, X_{0}=0$. (Note that for convenience the indices have been changed.)

Thus $Y(\tau)$ satisfies $(*)_{n}$ for $n \neq 1, m_{2}, \ldots, m_{l}$ and so, by the implicit function theorem, there is a neighborhood of $\left(X_{j}=0, j=1, \ldots, l, \mu=0, \nu=\beta\right)$ in $\mathbf{C}^{M l} \times \mathbf{R}^{k}$ such that $(*)_{n}, n \neq 1, m_{2}, \ldots, m_{l}$, has a unique solution $Y\left(X_{1}, \ldots, X_{l}, \mu, \nu, \tau\right)$. This solution is $C^{1}$ in $X_{1}, \ldots, X_{l}$, continuous in $\mu, \nu$, belongs to $B$ and

$$
\|Y(\tau)\|_{B}=o\left(\left\|\left(X_{1}, \ldots, X_{l}\right)\right\|\right) \quad \text { uniformly in } \mu, \nu .
$$

(This reduction process is standard. One could have reduced further to the kernels of $i \beta m_{j}-L(0)$ but this is unnecessary for the moment. See [I1, I2, or FR] for a similar reduction. This new reduction will be done in Remark II.3.4 in §II.4.)

The problem is then equivalent, in this neighborhood of $(0,0, \beta)$, to the finite set of bifurcation equations

$$
\left(\text { ivm }_{j} I-L(\mu)\right) X_{j}-g_{j}\left(\mu, \nu, X_{1}, \ldots, X_{l}\right)=0, \quad j=1, \ldots, l,
$$

with

$$
g_{j}\left(\mu, \nu, X_{1}, \ldots, X_{l}\right)=\frac{1}{2 \pi} \int_{0}^{2 \pi} g\left(\mu, \sum_{-l}^{l} X_{j} e^{i m_{j} \tau}+Y\left(X_{1}, \ldots, X_{l}, \mu, \nu, \tau\right)\right) e^{-i m_{j} \tau} d \tau
$$

This will be written as

$(*)_{h_{j}}$

$$
B_{j}(\lambda) X_{j}-g_{j}(\lambda, X)=0, \quad j=1, \ldots, l,
$$

or

$$
B(\lambda) X-g(\lambda, X)=0 .
$$

Here $\lambda$ stands for the vector $(\mu, \nu-\beta)$ in $\mathbf{R}^{k}$.

REMARK II.1. It is clear that the same derivation is possible if the equation (*) were complex valued from the start. Then one has to consider the full Fourier series and, for technical reasons, to look at the conjugates of $(*)_{n}$ for $n$ negative: for the real case this amounts to twice considering the same problem. Also one could have 
phrased the problem in terms of real Fourier series but then the group action, which will be seen to consist in commutation with complex numbers, is translated into commutation with $2 \times 2$ matrices. These are more difficult to handle, although everything else goes through, and in any case force a complex structure on the problem.

II.2. Study of the bifurcation equations.

II.2.A. Basic properties.

(a) $B_{j}(\lambda)$ belongs to $\mathrm{GL}\left(\mathrm{C}^{M}\right)$ for $\lambda \neq 0, \lambda$ small.

(b) $g_{j}(\lambda, X)=o(\|X\|)$ for $X$ small.

(c) For each $\rho$ small enough, there is an $r$ such that $(*)_{b}$ has no solution with $\|\lambda\|=\rho, 0<\|X\| \leqslant r$ (this follows easily from (a) and (b)). In this section it will be proved that $(*)_{b}$ has a solution with $\|\lambda\| \leqslant \rho,\|X\|=r(\rho)$ for every $\rho$ small enough.

$$
g_{j}\left(\lambda, e^{i \phi} X_{1}, e^{i m_{2} \phi} X_{2}, \ldots, e^{i m_{l} \phi} X_{l}\right)=e^{i m_{,} \phi} g_{j}\left(\lambda, X_{1}, \ldots, X_{l}\right) .
$$

This is the important equivariance property of the bifurcation equation and comes from the autonomous character of $(*)$, since it relates the bifurcation equation for $X(\tau+\phi)$ to the equation for $X(\tau)$, both functions describing the same orbit.

Proof of (d). If

$$
g_{n}\left(\mu, X_{\phi}\right) \equiv \frac{1}{2 \pi} \int_{0}^{2 \pi} g(\mu, X(\tau+\phi)) e^{-i n \tau} d \tau,
$$

then, by change of variables and periodicity, one has $g_{n}\left(\mu, X_{\phi}\right)=e^{i n \phi} g_{n}\left(\mu, X_{0}\right)$. Hence, by the uniqueness of $Y$, one has that

$$
Y\left(e^{i \phi} X_{1}, \ldots, e^{i m_{l} \phi} X_{l}, \mu, \nu, \tau\right)=Y\left(X_{1}, \ldots, X_{l}, \mu, \nu, \tau+\phi\right) .
$$

Then, from the definition of $g_{j}$ and the above property of Fourier coefficients, one has the result. Q.E.D.

II.2.B. Extension of maps. Choosing $\rho$ and $r$ as in property (c), one wishes to find solutions to $(*)_{b}$ in the set $B^{k} \times S^{2 / M-1}$ of $(\lambda, X)$ with $\|\lambda\| \leqslant \rho,\|X\|=r$, knowing that $(*)_{b}$ maps $S^{k-1} \times S^{2 / M-1}$ into $C^{I M}-\{0\}$. From the topological point of view, one would like an answer to the stronger question: Does any equivariant extension of $(*)_{b}$ from $S^{k-1} \times S^{2 / M-1}$ have a zero or, conversely, does there exist an equivariant extension of $(*)_{b}$ without zeros? Without loss of generality one may divide $(*)_{b}$ by its norm $/ r$, on $S^{k-1} \times S^{2 / M-1}$, and assume that $(*)_{b}$ has its range in $S^{2 l M-1} \equiv\left\{Y \in C^{l M},\|Y\|=r\right\}$. (If $(*)_{b}$ has an extension $F(\lambda, X)$, then $r(*)_{b} /\left\|(*)_{b}\right\|$ has the extension $r F(\lambda, X) /\|F(\lambda, X)\|$. Conversely, if $r(*)_{b} /\left\|(*)_{b}\right\|$ has the extension $G(\lambda, X)$, then $F(\lambda, X)=r^{-1} G(\lambda, X)\left(\rho-\|\lambda\|+\|\lambda\|\left\|(*)_{b}(\lambda \rho /\|\lambda\|, X)\right\| / \rho\right)$ is an extension for $(*)_{b}$. Since the group action is an isometry, the positive factors are invariant and the equivariance is preserved.)

II.2.C. Nonequivariant extensions. Now continuous (real) extensions are treated using obstruction theory. In the present case one would get obstructions in $H^{q+1}\left(B^{k} \times S^{2 / M-1}\right), S^{k-1} \times S^{2 l M-1}, \Pi_{q}\left(S^{2 l M-1}\right)$ : these cohomology groups are nontrivial only for $q=k-1$ and $q=k+2 l M-2$. In both cases they are equal to the coefficient group $\Pi_{q}\left(S^{2 / M-1}\right)$ (the main ideas and results of obstruction theory 
will be summarized in the next section). The primary obstruction is the class of $B(\lambda) X_{0}\left(X_{0}\right.$ a fixed point in $\left.S^{2 / M-1}\right)$ : its nonvanishing gives bifurcation in all directions $X_{0}$.

From the exact homotopy sequence

$$
\rightarrow \Pi_{k-1}\left(U_{I M-1}\right) \stackrel{i_{*}}{\rightarrow} \Pi_{k-1}\left(U_{I M}\right) \stackrel{P_{*}}{\rightarrow} \Pi_{k-1}\left(S^{2 / M-1}\right) \rightarrow
$$

for the fiber bundle for unitary groups

$$
U_{I M-1} \stackrel{i}{\rightarrow} U_{I M} \stackrel{P}{\rightarrow} S^{2 / M-1}
$$

(see [St] or [I4, §III.1]), $P_{*}[B(\lambda)] \equiv\left[B(\lambda) X_{0}\right]=0$ if and only if $[B(\lambda)]$ belongs to Image $i_{*}$, that is if and only if $B(\lambda)$ can be deformed to a family of matrices of the form

$$
\left(\begin{array}{cc}
I & 0 \\
0 & C(\lambda)
\end{array}\right)
$$

with $C(\lambda)$ in $U_{I M-1}$.

The second obstruction turns out to be the class of $\left(C(\lambda) \bar{X}, \rho^{2}-2\|\lambda\|^{2}\right)$ in $\Pi_{k+2 / M-1}\left(S^{2 / M-1}\right)$, that is the image under Whitehead's $J$-homomorphism of $C(\lambda)$. Its suspension, $\left(B(\lambda) X,\|X\|^{2}-r^{2}\right)$, gives solutions with $\|X\|=r$ and was studied in [AY1, 13, 14]. The derivation of the above result and other relations between these invariants will be given in another article. ${ }^{3}$ Here it is enough to remark that with this approach (ignoring the equivariance) one gets the analogue of Theorem 1.

II.2.D. Equivariant extensions. In order to take account of the action of the group, that is of the phase translation along orbits, it is necessary to look at the graph map $S^{k-1} \times S^{2 / M-1} \rightarrow S^{k-1} \times S^{2 / M-1} \times S^{2 / M-1}$, given by $(\lambda, X) \rightarrow\left(\lambda, X,(*)_{b}(\lambda, X)\right)$, where $(*)_{b}$ has been normalized. Then if one defines the group action as

$$
e^{i \phi} X \equiv\left(e^{i \phi} X_{1}, \ldots, e^{i m_{l} \phi} X_{l}\right)
$$

it is clear that $\left(\lambda, e^{i \phi} X\right)$ is sent into $\left(\lambda, e^{i \phi} X, e^{i \phi}(*)_{b}(\lambda, X)\right)$ and that it is enough to know the value of this map at one point of the orbit. This implies that, taking the quotient on both sides by the action, one will get a continuous map and conversely. However, due to the fact that the action is not free $\left(\left(0, \ldots, 0, X_{l}\right)\right.$ for example has an isotropy group $\left.\phi= \pm 2 k \pi / m_{l}, k=0,1, \ldots, m_{l}-1\right)$, the quotient space $S^{2 l M-1} / S^{1}$ is a stratified set and obstruction theory is not readily applicable (see however [Ko, Proposition 2.12]). Thus the free case will be studied first $(l=1$, no "weak" resonance) and then the general case will be reduced to the free case by a trick which will partly explain the change in the Fuller's degree.

In the free case, $l=1$, the quotient of $S^{2 M-1}$ by the standard action is the complex projective space $\mathbf{C} P^{M-1}$ and the quotient of $S^{2 M-1} \times S^{2 M-1}$ by the free action $e^{i \phi}(X, Y)=\left(e^{i \phi} X, e^{i \phi} Y\right)$ is denoted by $S^{2 M-1} \cdot \times{ }_{S^{1}} S^{2 M-1}$. Now, if one considers the sphere bundle

$$
S^{2 M-1} \rightarrow S^{k-1} \times S^{2 M-1} \times S^{1} S^{2 M-1} \stackrel{P}{\rightarrow} S^{k-1} \times \mathrm{C} P^{M-1}
$$

\footnotetext{
${ }^{3}$ There is a hint that obstruction theory is relevant to bifurcation in [A].
} 
with unitary bundle group and $P(\lambda,[X, Y])=(\lambda,[X])$, it is easy to see that cross-sections of this bundle, i.e. maps $\psi(\lambda,[X])=(\lambda,[X, F(\lambda, X)])$, are in one-to-one correspondence with equivariant maps $F(\lambda, X)$ from $S^{k-1} \times S^{2 M-1}$ into $S^{2 M-1}$ (see [Br, Chapter II, 2.6]). Then one has to extend the map induced by $(*)_{b}$ to a continuous cross-section of the bundle, with $S^{k-1}$ replaced by $B^{k}$, a situation in which one may apply obstruction theory.

\section{II.3. Facts about obstruction theory.}

For the reader's convenience the main ideas and results needed in this paper will be recalled.

Consider a sphere bundle over a finite cell complex $K: S^{n-1} \rightarrow \mathscr{B} \rightarrow K$ with connected group bundle (so that the coefficient bundle is a product [St, 30.4, p. 153]). Let $L$ be a subcomplex of $K$. Recall that a cell complex $K$ is $\cup \sigma_{i}^{q}$, $q=0, \ldots, N=\operatorname{dim} K, i=1, \ldots, \alpha_{q}$, such that (i) $\sigma_{i}^{q}$ is a $q$-cell, (ii) if $K^{p}=\bigcup_{q \leqslant p} \sigma_{i}^{q}$ is the $q$ th skeleton of $K$, then $\sigma_{i}^{q} \cap K^{q-1}=\partial \sigma_{i}^{q}$ is the exact union of cells (called the faces of $\sigma_{i}^{q}$ ), (iii) if $i \neq j$, then Int $\sigma_{i}^{q} \cap$ Int $\sigma_{j}^{q}=\varnothing$. Cells are defined as the images of the unit ball under an "attaching map" which is a homeomorphism on the interior.

If $f$ is a cross-section of the bundle over $L$, then one tries to extend $f$ to a full cross-section by extending it to $\bar{K}^{q} \equiv K^{q} \cup L$ and increasing the dimension $q$. (Since $K^{0}$ is a collection of points it is always possible to have an extension to $\bar{K}^{0}$ ). If $f$ has been extended to $\bar{K}^{q}$, one has to extend it over all $(q+1)$ cells of $K-L$. But if $\sigma^{q+1}$ is such a cell, then, since $\sigma$ is contractible, it is possible to assume that $\mathscr{B}$ over $\sigma$ is just a product bundle and that $f \mid \partial \sigma$ is of the form $(x, g(x))$, with $g(x)$ : $\partial \sigma \rightarrow S^{n-1}$. But then it is well known that $g(x)$ is extendible to a map from $\sigma$ into $S^{n-1}$ if and only if $g$ is deformable to a constant map in $\Pi_{q}\left(S^{n-1}\right)$ (see [I4] for example). Since several $(q+1)$ cells may be attached to the boundary cells, one gets in fact a cochain $C^{q+1}(f)$ in $C^{q+1}\left(K, L ; \Pi_{q}\left(S^{n-1}\right)\right)$, which is a cocycle with the following properties:

(1) If $f_{0}$ and $f_{1}$ are two cross-sections of the bundle over $\bar{K}^{q}$ which are homotopic, then $C^{q+1}\left(f_{0}\right)=C^{q+1}\left(f_{1}\right)$ [Hu, Lemma 3.3, p. 177].

(2) If $f_{0}$ is as above, then $f_{0}$ extends to $\bar{K}^{q+1}$ if and only if $C^{q+1}\left(f_{0}\right)=0[\mathbf{H u}$, Lemma 3.2, p. 177].

(3) If $f$ is a cross-section of $\mathscr{B} \mid \bar{K}^{q-1}$ and $f$ is extendible over $\bar{K}^{q}$, then the set of all $(q+1)$-dimensional obstruction cocycles form a single cohomology class $\gamma\left(f_{q}\right)$ in $H^{q+1}\left(K, L ; \Pi_{q}\left(S^{n-1}\right)\right)$ and $f$ extends over $\bar{K}^{q+1}$ if and only if $\gamma\left(f_{q}\right)=0$ (i.e. one may have to modify the previous extension but only on $q$-cells, keeping fixed the extension on $\bar{K}^{q-1}$ ) [Hu, 5.1, p. 180; St, 34.2, p. 174].

(4) If $\theta^{q+1}(f) \subset H^{q+1}\left(K, L ; \Pi_{q}\left(S^{n-1}\right)\right)$ is defined as empty if $f$ is not extendible to $\bar{K}^{q}$ and as the set of all $\gamma^{q+1}\left(f_{q}\right)$ for all extensions $f_{q}$ of $f$ (cross-section of the bundle over $L$ ) to cross-sections over $\bar{K}^{q}$, then this obstruction set has the following properties: (i) homotopic maps (as sections over $L$ ) have the same obstruction set; (ii) $f$ is extendible to $\bar{K}^{q}$ if and only if $\theta^{q+1}(f) \neq \varnothing$; (iii) $f$ is extendible to $\bar{K}^{q+1}$ if and only if 0 belongs to $\theta^{q+1}(f)([\mathbf{H u}, 6.1$, p. 181, 6.4 and 6.5, p. 182; St, 34.9, p. 176] use the homotopy lifting property). 
(5) If $q=n-1$, then the obstruction set $\theta^{n}(f)$ reduces to a single element, the primary obstruction, $\gamma^{n}(f)=\delta \kappa^{n-1}(f)$ where

$$
\delta: H^{n-1}\left(L ; \Pi_{n-1}\left(S^{n-1}\right)\right) \rightarrow H^{n}\left(K, L ; \Pi_{n-1}\left(S^{n-1}\right)\right)
$$

is the usual coboundary operator in the cohomology sequence for the pairs $(K, L)$ and $\kappa^{n-1}(f)$ is the characteristic element of $f$ (given by $f^{*}\left(\kappa^{n-1}\left(S^{n-1}\right)\right)$ ) with

$$
f^{*}: H^{n-1}\left(S^{n-1} ; \Pi_{n-1}\left(S^{n-1}\right)\right) \rightarrow H^{n-1}\left(L ; \Pi_{n-1}\left(S^{n-1}\right)\right)
$$

and $\kappa^{n-1}\left(S^{n-1}\right)$ is the generator of the first group) [Hu, 13.1, pp. 189, 193; St, 35.4, p. 178].

(6) Finally one has obstructions for lifting homotopies (with $K$ replaced by $K \times I)$ : If $f_{0}$ and $f_{1}$ are cross-sections of $\mathscr{B} \mid \bar{K}^{q}$ and if $h_{t}$ is a homotopy of $f_{0} \mid \bar{K}^{q-1}$ to $f_{1} \mid \bar{K}^{q-1}$, then one has a difference cochain $d^{q}\left(f_{0}, h_{t}, f_{1}\right)$ in $C^{q}\left(K, L ; \Pi_{q}\left(S^{n-1}\right)\right)$ such that

(a) $\delta d^{q}\left(f_{0}, h_{t}, f_{1}\right)=C^{q+1}\left(f_{0}\right)-C^{q+1}\left(f_{1}\right), \delta$ the coboundary operator [Hu, Lemmas 4.1, 4.2, p. 179].

(b) $d^{q}\left(f_{0}, h_{t}, f_{1}\right)=0$ if and only if $h_{t}$ extends to a homotopy of $f_{0} \mid \bar{K}^{q}$ to $f_{1} \mid \bar{K}^{q}[\mathbf{S t}$, 33.4, p. 171].

(c) If $f$ and $g$ are cross-sections of $\mathscr{B}$ (or of $\mathscr{B}$ over $\bar{K}^{q+1}$ ), such that $f=g$ on $L$, and if $h_{t}$ is a homotopy, relative to $L$, of $f \mid \bar{K}^{q-2}$ to $g \mid \bar{K}^{q-2}$ which is extendible to a homotopy $h_{t}^{\prime}$, relative to $L$, of $f \mid \bar{K}^{q-1}$ to $g \mid \bar{K}^{q-1}$, then the set of obstruction cocycles $d^{q}\left(f, h_{t}^{\prime}, g\right)\left(C^{q+1}(f)=C^{q+1}(g)=0\right)$ form a single cohomology class $\delta^{q}\left(f, h_{t}, g\right)$ in $H^{q}\left(K, L ; \Pi_{q}\left(S^{n-1}\right)\right)$ and $h_{t}$ is extendible to a homotopy of $f \mid \bar{K}^{q}$ to $g \mid \bar{K}^{q}$ if and only if $\delta^{q}\left(f, h_{t}, g\right)=0$ [St, 34.6, p. 175; Hu, 8.3, p. 184] (i.e. one may have to modify $h_{t}^{\prime}$ on the $(q-1)$ cells). As before one may define obstruction sets, however only the primary obstruction will be needed, i.e. for $q=n-1$ : then, from the above, $f$ and $g$ are always homotopic on $\bar{K}^{n-2}$, the obstruction set consists of a single element $\delta^{n-1}(f, g)$ in $H^{n-1}\left(K, L ; \Pi_{n-1}\left(S^{n-1}\right)\right)$ with $j^{*} \delta^{n-1}(f, g)=\kappa^{n-1}(f)-$ $\kappa^{n-1}(g)$, where $j^{*}$ is the homomorphism $H^{n-1}(K, L) \stackrel{j^{*}}{\rightarrow} H^{n-1}(K)$ induced by inclusion and $\kappa^{n-1}(f)$ is the characteristic element of $f$ (as a mapping defined on $K$ ).

II.4. The free action case.

II.4.A. The cohomology groups. Eventual obstructions in this case, as explained in the previous sections, will be elements of the cohomology groups

$$
H^{q+1}\left(B^{k} \times \mathbf{C} P^{M-1}, S^{k-1} \times \mathbf{C} P^{M-1} ; \Pi_{q}\left(S^{2 M-1}\right)\right) .
$$

Now $C P^{M-1}$, an $M-1$ complex manifold, has the following cell structure:

$$
C P^{M-1}=B^{0} \cup B^{2} \cup \cdots \cup B^{2 M-2}=C P^{M-2} \cup B^{2 M-2}
$$

where the cell

$$
B^{2 M-2}=\left\{\left(x_{1}, \ldots, x_{M-1}\right) \in \mathbf{C}^{M-1} / \sum_{i=1}^{M-1}\left|x_{i}\right|^{2} \leqslant r^{2}\right\}
$$

is sent into $\left[x_{1}, \ldots, x_{M-1},\left(r^{2}-\sum_{i=1}^{M-1}\left|x_{i}\right|^{2}\right)^{1 / 2}\right]$ (so that $\partial B^{2 M-2}$ is sent into $\left[x_{1}, \ldots, x_{M-1}, 0\right]$, considered as the embedding of $C P^{M-2}$ into $C P^{M-1}$ [Sp, p. 146]). 
$B^{k} \cup S^{k-1}$ is given the usual equatorial cell decomposition. This implies that the cells in $K-L$ are of the form $B^{k} \times B^{2 p}, p=0, \ldots, M-1$. Since, for a given $p$, there is just one cell and no cells in dimension $2 p+k \pm 1$, then all cochains are cocycles and there are no coboundaries. Thus

$$
\begin{gathered}
H^{k+2 p}(K, L)=Z, \quad p=0, \ldots, M-1, \\
H^{q+1}(K, L)=0 \text { for } q+1 \text { different from the above values. }
\end{gathered}
$$

REMARK II.4. Another way to see this, since only the $q$ 's with $q \geqslant 2 M-1$ are of interest, is to consider the exact sequence

$$
\begin{aligned}
H^{q}\left(B^{k} \times \mathbf{C} P^{M-1}\right) & \rightarrow H^{q}\left(S^{k-1} \times \mathbf{C} P^{M-1}\right) \rightarrow H^{q+1}(K, L) \\
& \rightarrow H^{q+1}\left(B^{k} \times \mathbf{C} P^{M-1}\right) .
\end{aligned}
$$

Since $B^{k}$ is contractible, $H^{l}\left(B^{k} \times \mathbf{C} P^{M-1}\right)=H^{l}\left(\mathbf{C} P^{M-1}\right)(=0$ for $l \geqslant 2 M-1$, from the dimension), then

$$
H^{q+1}(K, L) \cong H^{q}\left(S^{k-1} \times \mathbf{C} P^{M-1}\right) \text { for } q \geqslant 2 M-1 .
$$

From Künneth's formula and the restriction on $q$, this last group is $H^{k-1}\left(S^{k-1}\right)$ $\otimes H^{q-k+1}\left(\mathbf{C} P^{M-1}\right)$ (there is no contribution from $H^{0}\left(S^{k-1}\right)$ ). Since $H^{2 p}\left(\mathbf{C} P^{M-1}\right)$ $=Z, H^{2 p+1}\left(\mathbf{C} P^{M-1}\right)=0,0 \leqslant p \leqslant 2 M-2$, one gets the same result.

II.4.B. Deformation to the linear part. The primary obstruction will be for $p=0$, if $k=q+1 \geqslant 2 M$, in $\Pi_{k-1}\left(S^{2 M-1}\right)$ (by the universal coefficient formula); for $p=$ $(2 M-k) / 2$, if $k$ is even and $k+2 p=q+1=2 M$ (so that $k \leqslant 2 M$ ), in $Z$; or for $p=(2 M-1-k) / 2$, if $k$ is odd and $k+2 p=q+1=2 M+1$, in $\Pi_{2 M}\left(S^{2 M-1}\right)$ $=Z_{2}$. However from properties (4) and (5), homotopic maps on $L$ have the same obstruction set (reduced to one element for the primary obstruction) and, since homotopic sections are in one-to-one correspondence with equivariant homotopic maps from $S^{k-1} \times S^{2 M-1}$ into $C^{M}-\{0\}$, one may deform the nonlinear part of $(*)_{b}$ to 0 . It is enough to consider the obstruction sets for $B(\lambda) X$ or for any $D(\lambda) X$ if $B(\lambda)$ is deformable in $\Pi_{k-1}\left(G L\left(C^{M}\right)\right.$ ) to $D(\lambda)$ (equivariance for linear maps corresponds to complex matrices). One may use the following periodicity result:

TheOREM (BOTT). If $k \leqslant 2 M$, then $\Pi_{k-1}\left(\mathrm{GL}\left(\mathrm{C}^{M}\right)\right)$ is 0 if $k$ is odd and $Z$ if $k$ is even. So that if $k$ is odd, any matrix $B(\lambda)$ is deformable to $I$ and, if $k$ is even, there is an integer, Degree $B(\lambda)$, such that $B(\lambda)$ is homotopic to $I$ if and only if this Degree is $0[$ At $]$.

But fiom the exact sequence

$$
\Pi_{k}\left(S^{2 M-1}\right) \stackrel{\delta}{\rightarrow} \Pi_{k-1}\left(U_{M-1}\right) \stackrel{i_{*}}{\rightarrow} \Pi_{k-1}\left(U_{M}\right) \stackrel{P_{*}}{\rightarrow} \Pi_{k-1}\left(S^{2 M-1}\right)
$$

for the fiber bundle of unitary groups, one has that, for $k<2 M-1, i_{*}$ is an isomorphism (so that $\Pi_{k-1}\left(U_{M}\right) \cong \Pi_{k-1}\left(U_{M+1}\right)$ for $k \leqslant 2 M$ ) and, for $k=2 s \leqslant$ $2 M, B(\lambda)$ is deformable to $\left(\begin{array}{c}I \\ 0\end{array} C_{(\lambda)}^{0}\right)$ with $C(\lambda)$ in $\mathrm{GL}\left(\mathbf{C}^{s}\right)$. Furthermore $P_{*}$, as a mapping from $\Pi_{2 s-1}\left(U_{s}\right)$ into $\Pi_{2 s-1}\left(S^{2 s-1}\right)$ (both equal to $Z$ ), sends the generator into an element of degree $(s-1)$ !. (Recall that $P_{*} B(\lambda)=\left[B(\lambda)(1,0,0, \ldots, 0)^{T}\right]$.) 
Then Degree $B(\lambda) \equiv(-1)^{s-1}$ degree $\left(P_{*} C(\lambda)\right) /(s-1)$ ! [At]. If $k=2, B(\lambda)$ is deformable to $D(\lambda)$ if and only if $\operatorname{det} B(\lambda)$ is deformable to det $D(\lambda)$ as mappings from $S^{1}$ into $C-\{0\}$ and Degree $B(\lambda)=$ degree det $B(\lambda)$. (Since $C(\lambda)$ is a complex number and $B(\lambda)$ is deformable to such a matrix, det $B(\lambda)$ is deformable to $C(\lambda)$. This implies that one can replace $C(\lambda)$ by det $B(\lambda)$.)

II.4.C. The primary obstruction. It remains to compute the obstruction: let $X$ be written as $\tilde{X} \oplus X_{0}$, with $\tilde{X}$ in $\mathbf{C}^{P}$ and $\tilde{X}=0$, if $k \geqslant 2 M$. Consider $\mathbf{C} P^{M-1}=\mathbf{C} P^{p-1}$ $\cup B^{2 p} \cup \cdots \cup B^{2 M-2}$ with

$$
\mathbf{C} P^{p-1}=\left\{\left[x_{1}, \ldots, x_{p}, 0, \ldots, 0\right] \equiv[\tilde{X}, 0, \ldots, 0]\right\} \subset \mathbf{C} P^{M-1}
$$

(if $p=0, \mathbf{C} P^{-1}$ is taken as empty).

If $k$ is odd and $k \leqslant 2 M$, then $(*)_{b}$ is homotopic to $X$ which is extendible to $K$, so all obstructions vanish. Thus it is enough to study the cases $p=0$ if $k \leqslant 2 M$, and $p=M-s$ if $k \equiv 2 s \leqslant 2 M$.

Define $C(\lambda)$ for $\|\lambda\| \leqslant \rho$ by $\|\lambda\| \rho^{-1} C\left(\lambda \rho /\|\lambda\|^{-1}\right)$. Then the section $\left(\lambda,\left[\tilde{X}, X_{0}\right]\right) \rightarrow$ $\left(\lambda,\left[\tilde{X}, X_{0}, \tilde{X}, C(\lambda) X_{0}\right]\right)$ is already defined on $L \cup K^{k+2 p-1} \equiv \bar{K}^{k+2 p-1}$, since the $(k+2 p-j)$ cells of $K-L, j \geqslant 1$, are of the form $B^{k} \times B^{2 p-j}$ for $j$ even: on them $X_{0}$ is 0 . On the single cell $B^{k} \times B^{2 p}$, attached by $\{(\lambda, \tilde{X}) /\|\lambda\| \leqslant \rho,\|\tilde{X}\| \leqslant r\}$ $\rightarrow\left(\lambda,\left[\tilde{X},\left(r^{2}-\|\tilde{X}\|^{2}\right)^{1 / 2}, 0, \ldots, 0\right]\right)$, the section looks like

$$
\left(\lambda,\left[\tilde{X},\left(r^{2}-\|\tilde{X}\|^{2}\right)^{1 / 2}, 0, \ldots, \tilde{X}, C(\lambda)\left(\left(r^{2}-\|\tilde{X}\|^{2}\right)^{1 / 2}, 0, \ldots, 0\right)^{T}\right]\right) .
$$

Now if one defines $\phi(\lambda, \tilde{X}, Y)=\left(\lambda,\left[\tilde{X},\left(r^{2}-\|\tilde{X}\|^{2}\right)^{1 / 2}, 0, \ldots, 0, Y\right]\right)$ from $B^{k} \times$ $B^{2 p} \times S^{2 M-1}$ into the total space of the bundle over the cell $B^{k} \times B^{2 p}$, and $P_{2}\left(\lambda,\left[\tilde{X},\left(r^{2}-\|\tilde{X}\|^{2}\right)^{1 / 2}, 0, \ldots, Y\right]\right)=Y$ (note that the phase is fixed by choosing the second component real), then $\phi$ is clearly a trivialization of the bundle and the obstruction is the class of the map

$$
(\lambda, \tilde{X}) \rightarrow\left(\tilde{X}, C(\lambda)\left(\left(r^{2}-\|\tilde{X}\|^{2}\right)^{1 / 2}, 0, \ldots, 0\right)^{T}\right),
$$

as a mapping from the boundary of $B^{k} \times B^{2 p}$ into $S^{2 M-1}$. (Note that for $C(\lambda)$ unitary, $\|Y\|=r$; this is also the reason for taking the square root of $r^{2}-\|\tilde{X}\|^{2}$.)

Now this map is deformable to $\left(\tilde{X}, C(\lambda)(1,0, \ldots, 0)^{T}\right)$. Hence the class of the application $(\tilde{X}, \lambda) \rightarrow\left(\tilde{X}, C(\lambda)(1,0, \ldots, 0)^{T}\right)$ (using the degree of product for the case $p=M-s)$ is the class of $C(\lambda)(1,0, \ldots, 0)^{T}$ in $\Pi_{k-1}\left(S^{2 M-1}\right)$ (if $\left.p=0\right)$ or in $\Pi_{2 s-1}\left(S^{2 s-1}\right)$ (if $p=M-s$ ). (Since $\tilde{X}$ is complex, the mapping $(\lambda, \tilde{X}) \rightarrow(\tilde{X}, \lambda)$ has degree 1.)

In conclusion, one has the following result:

THEOREM II.4.1. The primary obstruction is:

(i) 0 if $k$ is odd and $k \leqslant 2 M$, all obstructions vanish.

(ii) $(-1)^{s-1}(s-1)$ ! Degree $B(\lambda)$ if $k=2 s \leqslant 2 M$. If Degree $B(\lambda)=0$, then all other obstructions vanish $(B(\lambda)$ is deformable to $I)$.

(iii) The class of $B(\lambda) X_{0}$, with $X_{0}$ fixed, if $k \geqslant 2 M$ in $\Pi_{k-1}\left(S^{2 M-1}\right)$. If this class is nontrivial, then one has bifurcation in all directions: in fact taking any $X_{0}$ fixed, with $\left\|X_{0}\right\|=r$, then $B(\lambda) X_{0}-g\left(\lambda, X_{0}\right)$ is nontrivial in $\Pi_{k-1}\left(S^{2 M-1}\right)$ and the usual result applies (see [I1]). 
Note also that one could look at the class of the pair $\left(B(\lambda) X-g(\lambda, X), X-X_{0}\right)$ from the sphere $\left\{(\lambda, X) /\|\lambda\|^{2}+\|X\|^{2}=\rho^{2}+r^{2}\right\}$ into $\mathbf{R}^{4 M}-\{0\}$, which is the $2 M$ suspension of the preceding: in this case one is fixing the phase; see [14]. (This suspension is an isomorphism if $k<4 M-2$.) See however Remark II.4.4 below. Before computing $n(2)$, the following comments may be useful.

REMARKS. II.4.a. If $B(\lambda)$ is deformable to $I$ via $B(\lambda, t)$ for $\|\lambda\|=\rho$, then $B(\lambda \rho /\|\lambda\|,\|\lambda\| / \rho)\left(\|\lambda\| / \rho+\|X\|^{2}\right) X$ is an equivariant extension of $B(\lambda)=B(\lambda, 1)$ for $\|\lambda\|=\rho$, without nontrivial zeros. However in the case of the Hopf bifurcation problem the dependency on $\lambda$ should be of the form $i \nu I-L(\mu)$.

II.4.b. Step-by-step construction of the extension. One can get to this point without using quotients, cross-sections or the heavy machinery of obstruction theory, but considering directly the problem of equivariant extensions of mappings from $S^{k-1}$ $\times S^{2 M-1}$ into $S^{2 M-1}$ to mappings from $B^{k} \times S^{2 M-1}$ into $S^{2 M-1}$ : in fact if $G(\lambda, X)$, on $\{\|\lambda\|=1,\|X\|=1\}$, has norm 1 and $G\left(\lambda, e^{i \phi} X\right)=e^{i \phi} G(\lambda, X)$, then consider the following step-by-step equivariant extension: Let

$$
B^{2 p}=\left\{\left(x_{1}, \ldots, x_{p}, 0, \ldots, 0\right) \in \mathbf{C}^{M} /\left|x_{1}\right|^{2}+\cdots+\left|x_{p}\right|^{2} \leqslant 1\right\},
$$

$S^{2 p-1}$ its boundary. On $S^{1}, G\left(\lambda, x_{1}, 0, \ldots, 0\right)=e^{i \phi} G(\lambda, 1,0, \ldots, 0)$. As a mapping from $S^{k-1}$ into $S^{2 M-1}, G(\lambda, 1,0, \ldots, 0)$ extends to a mapping from $B^{k}$ to $S^{2 M-1}$ if and only if it is deformable to a constant map, i.e. is trivial in $\Pi_{k-1}\left(S^{2 M-1}\right)$. If this is the case, let $G_{0}(\lambda)$ be the extension and define $G_{0}\left(\lambda, x_{1}\right)=e^{i \phi} G_{0}(\lambda)$ for $x_{1}=e^{i \phi}$, which coincides clearly with the original map on $S^{k-1} \times S^{1}$. Let

$$
\tilde{G}_{1}\left(\lambda, x_{1}\right)= \begin{cases}G\left(\lambda, x_{1},\left(1-\left|x_{1}\right|^{2}\right)^{1 / 2}, 0, \ldots, 0\right) & \text { for }|\lambda|=1,\left|x_{1}\right| \leqslant 1 \\ G_{0}\left(\lambda, x_{1}\right) & \text { for }|\lambda| \leqslant 1,\left|x_{1}\right|=1 .\end{cases}
$$

$\tilde{G}_{1}\left(\lambda, x_{1}\right)$ is a continuous map from $\partial\left(B^{k} \times B^{2}\right)$ into $S^{2 M-1}$, which extends to $G_{1}\left(\lambda, x_{1}\right)$ as a mapping on $B^{k} \times B^{2}$, if and only if it is trivial in $\Pi_{k+1}\left(S^{2 M-1}\right)$. If this is so, define $\tilde{G}_{2}\left(\lambda, x_{1}, x_{2}\right)$ on $\partial\left(B^{k} \times B^{4}\right)$ as follows:

$$
\begin{aligned}
& G\left(\lambda, x_{1}, x_{2},\left(1-\left|x_{1}\right|^{2}-\left|x_{2}\right|^{2}\right)^{1 / 2}, 0, \ldots, 0\right) \text { for }|\lambda|=1,|X| \leqslant 1, \\
& G_{1}\left(\lambda, x_{1}\right) \text { for }|\lambda| \leqslant 1, x_{2}=\left(1-\left|x_{1}\right|^{2}\right)^{1 / 2}, \\
& e^{i \phi} G_{1}\left(\lambda, e^{-i \phi} x_{1}\right) \text { for }|\lambda| \leqslant 1, x_{2}=e^{i \phi}\left(1-\left|x_{1}\right|^{2}\right)^{1 / 2} .
\end{aligned}
$$

Note that if $x_{2}=0$, then $\left|x_{1}\right|=1$ and $G_{1}\left(\lambda, e^{-i \phi} x_{1}\right)=G_{0}\left(\lambda, e^{-i \phi} x_{1}\right)=e^{-i \phi} G_{0}(\lambda)$, so that the definition is consistent. Furthermore, it is easy to check that $\tilde{G}_{2}\left(\lambda, x_{1}, x_{2}\right)$ is equivariant on $B^{k} \times S^{3}$, so that $\tilde{G}_{2}\left(\lambda, x_{1}, x_{2}\right)=G\left(\lambda, x_{1}, x_{2}, 0, \ldots, 0\right)$ for $|\lambda|=1$, $|X|=1$, since both mappings are equivariant and coincide from the definition of $\tilde{G}_{1}\left(\lambda, x_{1}\right)$ for real positive $x_{2}$.

The obstruction for extension will be in $\Pi_{k+3}\left(S^{2 M-1}\right)$ and, if it vanishes, one has a map $G_{2}\left(\lambda, x_{1}, x_{2}\right)$ on $B^{k} \times B^{4}$. Then setting

$$
\tilde{G}_{3}\left(\lambda, x_{1}, x_{2}, x_{3}\right)=\left\{\begin{array}{l}
G_{2}\left(\lambda, x_{1}, x_{2}\right) \text { for }|\lambda| \leqslant 1, x_{3}=\left(1-\left|x_{1}\right|^{2}-\left|x_{2}\right|^{2}\right)^{1 / 2}, \\
e^{i \phi} G_{2}\left(\lambda, e^{-i \phi} x_{1}, e^{-i \phi} x_{2}\right) \text { for } x_{3}=e^{i \phi}\left(1-\left|x_{1}\right|^{2}-\left|x_{2}\right|^{2}\right)^{1 / 2},
\end{array}\right.
$$


one gets an equivariant map on $B^{k} \times S^{5}$, which coincides with

$$
G\left(\lambda, x_{1}, x_{2}, x_{3},\left(1-\sum_{i=1}^{3}\left|x_{i}\right|^{2}\right)^{1 / 2}, 0, \ldots, 0\right)
$$

on $S^{k-1} \times S^{5}$.

If one has an equivariant extension $\tilde{G}_{p}\left(\lambda, x_{1}, \ldots, x_{p}\right)$ on $B^{k} \times S^{2 p-1}$, then, by considering

$$
\begin{array}{lr}
G\left(\lambda, x_{1}, \ldots, x_{p},\left(1-\sum_{i=1}^{p}\left|x_{i}\right|^{2}\right)^{1 / 2}, 0, \ldots, 0\right) & \text { on } S^{k-1} \times B^{2 p}, \\
\tilde{G}_{p}\left(\lambda, x_{1}, \ldots, x_{p}\right) & \text { on } B^{k} \times S^{2 p-1},
\end{array}
$$

one has an obstruction in $\Pi_{k+2 p-1}\left(S^{2 M-1}\right)$ and, if it vanishes, an extension $G_{p}\left(\lambda, x_{1}, \ldots, x_{p}\right)$ to $B^{k} \times B^{p}$. Defining $\tilde{G}_{p+1}\left(\lambda, x_{1}, \ldots, x_{p+1}\right)$

$$
= \begin{cases}G_{p}\left(\lambda, x_{1}, \ldots, x_{p}\right) \quad \text { if } x_{p+1}=\left(1-\sum_{1}^{p}\left|x_{i}\right|^{2}\right)^{1 / 2} \\ e^{i \phi} G_{p}\left(\lambda, e^{-i \phi} x_{1}, \ldots, e^{-i \phi} x_{p}\right) & \text { if } x_{p+1}=e^{i \phi}\left(1-\sum_{1}^{p}\left|x_{i}\right|^{2}\right)^{1 / 2},\end{cases}
$$

one obtains an equivariant (and consistent) extension to $B^{k} \times S^{2 p+1}$, as above.

To finish the proof, one would need the equivariant homotopy extension property and to prove that the primary obstruction is unique and depends only on the equivariant homotopy class of $G(\lambda, X)$ on $S^{k-1} \times S^{2 M-1}$. This amounts to reproving these properties as done for $\mathbf{C} P^{M-1}$.

II.4.D. Computation of $n(2)$. The numbers $n_{j}(k)$ in the statement of the Main Theorem are just the Degrees of $i \nu m_{j} I-L(\mu)$ : To complete the proof of the local result (the existence of the continuum will be a consequence of the global result), it remains to compute $n(2)=$ degree $\operatorname{det} B(\lambda)$, with $B(\lambda)=\nu i I-L(\mu)$. Hence $B(\lambda)$ $=\Pi\left(\nu i-e_{j}(\mu)\right)$, where $e_{j}(\mu)$ are the eigenvalues of $L(\mu), j=1, \ldots, 2 M$ (which are continuous in $\mu) .|\nu-\beta|^{2}+|\mu|^{2}=\rho^{2}$, $\rho$ so small that if $e_{j}(\mu)-\nu i=0$, then $\mu=0$, $\nu=\beta$. It is enough to consider $e_{j}(\mu)$ corresponding to one of the eigenvalues passing through $i \beta$ : for the others the term $\nu i-e_{j}(\mu)$ can be deformed to $\beta i-e_{j}(0)$ (linearly on $(\mu, \nu-\beta)$ ) and then to 1 in $\mathbf{C}$. Then the degree of the determinant is the degree of $\Pi\left(\left(\nu-\beta-\gamma_{j}(\mu)\right) i-\alpha_{j}(\mu)\right)$, where $j=1, \ldots, d_{1}\left(d_{1}\right.$ the algebraic multiplicity of $i \beta), \alpha_{j}(\mu)$ vanishing only at $\mu=0 . \gamma_{j}(\mu)$ can be linearly deformed to 0 , $\alpha_{j}(\mu)$ can be linearly deformed to $\operatorname{Sign} \alpha_{j}(\mu)$ if it does not change sign, and then $(\nu-\beta) i-\operatorname{sign} \alpha_{j}(\mu)$ to 1 , or to $\pm \mu$ if $\alpha_{j}(\mu)$ changes sign as $\pm \mu$. The degree is then the degree of

$$
[(\nu-\beta) i-\mu]^{n_{+}}[(\nu-\beta) i+\mu]^{n_{-}}=i^{n_{+}+n_{-}} \lambda^{n_{+}} \bar{\lambda}^{n_{-}},
$$

where $\lambda=\nu-\beta+i \mu$. Since the factor $i^{n_{+}+n_{-}}$can be deformed to $1, \lambda$ contributes a winding number 1 and $\bar{\lambda}$ a winding number -1 ; the degree is $n_{+}-n_{-}=n$. Before 
treating the nonfree case in $\S I I .5$, the following comments go deeper into the study of the linear part.

REMARKS. II.4.1. Here one does not need any transversality argument as in [AY] or [I1, Appendix].

II.4.2. If one had considered the bifurcationequation $(*)_{1},(\bar{*})_{-1}$ for $\left(X_{1}, \bar{X}_{-1}\right)$ (the reason of conjugation is that the action on this couple is the standard action), then one would have had to compute the Degree of $((i \nu-L(\mu)),(\overline{-i \nu-L(\mu)}))$ which is, in the real case, twice the Degree of $i \nu-L(\mu)$, with the same result.

11.4.3. An example of a Hopf bifurcation with $k=4$.

$$
L\left(\mu_{1}, \mu_{2}, \mu_{3}\right)=\left[\begin{array}{cccc}
\mu_{1} & -\mu_{2} & -\beta & -\mu_{3} \\
-\mu_{2} & -\mu_{1} & \mu_{3} & -\beta \\
\beta & \mu_{3} & \mu_{1} & -\mu_{2} \\
-\mu_{3} & \beta & -\mu_{2} & -\mu_{1}
\end{array}\right] .
$$

It is easy to see that $\operatorname{det}(\lambda-L(\mu))=\lambda^{4}-2 \lambda^{2}\left(|\mu|^{2}-\beta^{2}\right)+\left(|\mu|^{2}+\beta\right)^{2}$ with $|\mu|^{2}$ $=\mu_{1}^{2}+\mu_{2}^{2}+\mu_{3}^{2}$, with eigenvalues $\pm\left(|\mu| \pm i \beta\right.$ ). (Note that if one takes $\mu_{2}=\mu_{3}=0$, then $n^{+}=n^{-}=0$, which is normal since the real parts of the eigenvalues, being continuous mappings from $\mathbf{R}^{k-1}-\{0\}$ into $\mathbf{R}-\{0\}$, cannot change sign if $k>2$.) If one considers the matrix

$$
P=\left[\begin{array}{cccc}
1 & 0 & 1 & 0 \\
0 & 1 & 0 & 1 \\
-i & 0 & i & 0 \\
0 & -i & 0 & i
\end{array}\right]
$$

then

$$
\begin{aligned}
& P^{-1}(L(\mu)-i \nu) P \\
& =\left[\begin{array}{cccc}
\mu_{1}+i(\beta-\nu) & -\mu_{2}+i \mu_{3} & 0 & 0 \\
-\mu_{2}-i \mu_{3} & -\mu_{1}+i(\beta-\nu) & 0 & 0 \\
0 & 0 & \mu_{1}-i(\beta+\nu) & -\mu_{2}-i \mu_{3} \\
0 & 0 & -\mu_{2}+i \mu_{3} & -\mu_{1}-i(\beta+\nu)
\end{array}\right]
\end{aligned}
$$

Since $P$ and $P^{-1}$ can be deformed to the identity as well as the lower right matrix (for $\nu$ close to $\beta$ ), the class of $L(\mu)-i \nu$ is the class of the matrix

$$
\left(\begin{array}{cc}
\mu_{1}+i(\beta-\nu) & -\left(\mu_{2}-i \mu_{3}\right) \\
-\left(\mu_{2}+i \mu_{3}\right) & -\left(\mu_{1}-i(\beta-\nu)\right)
\end{array}\right)
$$

and, by multiplying the second row by $e^{i \pi}$ (which is an admissible deformation), it is the class of

$$
\left(\begin{array}{cc}
\lambda_{1} & -\bar{\lambda}_{2} \\
\lambda_{2} & \bar{\lambda}_{1}
\end{array}\right) \text { with } \lambda_{1}=\mu_{1}+i(\beta-\nu), \lambda_{2}=\mu_{2}+i \mu_{3},
$$

denoted as $\lambda_{1} * \lambda_{2}$ in Atiyah's article, with Degree 1 . Note that replacing $\lambda_{2}$ by $\lambda_{2}^{n}$ or $\bar{\lambda}_{2}^{\prime \prime}$ one gets a matrix with Degree $n$ or $-n$, whereas for $k=2$, the Degree is limited by $\pm[M / 2]$, the maximum number of eigenvalues crossing at $i \beta$. 
II.4.4. Higher number of parameters. If one defines the product of two matrices as

$$
f(X) \tilde{*} g(Y)=\left(\begin{array}{cc}
f(X) \otimes 1_{N} & -1_{M} \otimes \bar{g}(Y) \\
-1_{M} \otimes g(Y) & -\bar{f}(X) \otimes 1_{N}
\end{array}\right),
$$

where $X \in \mathbf{R}^{N}, Y \in \mathbf{R}^{M}, f(X) \in \mathrm{GL}\left(\mathbf{C}^{M}\right)$ and $g(Y) \in \mathrm{GL}\left(\mathbf{C}^{N}\right)$, then one obtains an element of $\mathrm{GL}\left(\mathbf{C}^{2 M N}\right)$. The Degree of $f(X) \tilde{*} g(Y)$ is (Degree $f(X) \times$ Degree $g(Y)$ ) (this is the definition given in [At] with the negative of the second row). Then $\left(\mu_{1}+i \beta\right) \tilde{*} \lambda_{2} \tilde{*} \lambda_{3} \tilde{*} \ldots \tilde{*} \lambda_{s}$, with $\lambda_{j+1}=\mu_{2 j}+i \mu_{2 j+1}, j=1, \ldots, s-$ 1 , has Degree 1 (or Degree $\pm n$ if $\lambda_{2}$ is replaced by $\lambda_{2}^{n}$ or $\bar{\lambda}_{2}^{n}$ ) and, by construction, if $\mu_{1}=\lambda_{2}=\cdots=\lambda_{s}=0$, this is $i \beta I^{2^{s-1}}$. Write this matrix as $A+i B$ and consider the matrices

$$
\left(\begin{array}{cc}
A+i B & 0 \\
0 & A-i B
\end{array}\right) \equiv D, \quad P \equiv\left(\begin{array}{cc}
I & I \\
-i I & i I
\end{array}\right)
$$

Then

$$
L\left(\mu_{1}, \ldots, \mu_{2 s-1}\right) \equiv\left(\begin{array}{cc}
A & -B \\
B & A
\end{array}\right)=P D P^{-1}
$$

satisfies properties H.1-H.3, with eigenvalues $\pm i \beta$, and has Degree 1 (or Degree $\pm n)$. This implies that, for each even $k$, one has a matrix $L(\mu)$ acting on $\mathbf{R}^{M}$, with $M=2^{k / 2}$, with any Degree if $k \geqslant 4$, or with any possible Degree between $-[M / 2]$ and $[M / 2]$ if $k=2$.

II.4.5. Higher order differential equations. Note that one may also consider directly systems of higher order differential equations: after scaling the period, one has to work in $W^{p, 2}\left(S^{1}\right)^{M}, p$ the order of the system. For example, one may look at the system

$$
Y^{\prime \prime}+A_{0} Y+B(\mu) Y^{\prime}+C(\mu) Y=F\left(Y, Y^{\prime}, \mu\right)
$$

with $B(0)=C(0)=0, F$ a small nonlinearity. Writing $Y=\sum Y_{n} e^{i n \tau}$, one is lead to consider the system

$$
\left(-\nu^{2} n^{2} I+A_{0}+C(\mu)+i \nu n B(\mu)\right) Y_{n}-F_{n}\left(Y, Y^{\prime}, \mu\right)=0
$$

( $n \geqslant 0$ for real solutions as before), which is reducible to those $n$ 's such that $A_{0}-\nu^{2} n^{2} I$ is singular. For example, if $M=2, A_{0}=I, k=4$,

$$
B(\mu)=\left(\begin{array}{cc}
-\mu_{1} & \mu_{2} \\
\mu_{2} & \mu_{1}
\end{array}\right) \text { and } C(\mu)=\left(\begin{array}{cc}
0 & \mu_{3} \\
-\mu_{3} & 0
\end{array}\right)
$$

then, for $\nu$ close to 1 , one has to consider the above matrix for $n=1$, with determinant $\left(1-\nu^{2}\right)^{2}+\nu \mu_{1}^{2}+\mu_{2}^{2}+\mu_{3}^{2}$ (so that it is nonzero for $\lambda=(1-\nu, \mu) \neq$ $0)$. The obstruction is the class of $\left(\left(1-\nu^{2}\right) I+C(\mu)+i \nu B(\mu)\right)(1,0)^{T}$, that is of the vector $\left(1-\nu^{2}-i \nu \mu_{1},-\mu_{3}+i \mu_{2}\right)^{T}$. This is a mapping of degree 1 (use the product theorem), so that one has bifurcation. Note that if one tries to prove the existence of periodic solutions to this system by reducing the problem to one parameter $\mu_{j}=$ $\mu_{j}(\varepsilon), j=1,2,3$, then there will always be a nonlinearity without solutions. Choose a smooth path $\eta_{j}(t)$, transversal to the first at the origin, and replace $\mu$ by $\mu(\varepsilon)-$ $\eta\left(|Y|_{0}^{2}\right)$ in the matrices $B$ and $C$ : a periodic solution with $|Y|_{0}=r$ will give a solution to the linear system, which is possible only if $\mu=0$. But then $\mu(\varepsilon)$ and $\eta(t)$ are collinear (for $\varepsilon$ and $t$ small) only for $\varepsilon=t=|Y|_{0}^{2}=0$. 
II.4.6. Reduction to the kernel. The results above are valid for any matrix $B(\lambda)$ and most of them do not take into account the fact that $B(\lambda)=\nu i I-L(\mu)$ has the property that $B(0) \neq 0$.

THEOREM II.4.2. The primary obstruction, considered on $\mathbf{C}^{M}$, is always zero if $k>2 d$, where $d \equiv \operatorname{dim} \operatorname{ker} B(0)$.

Hence one has to consider the bifurcation equation on $\mathbf{C}^{d}$.

Proof. The equation $B(\lambda) X-g(\lambda, X)=0$ can be written as

$$
A X-T(\lambda) X-g(\lambda, X)=0
$$

with $A=B(0), T(\lambda)=B(0)-B(\lambda), T(0)=0$. Let $X=X_{1} \oplus X_{2}, X_{1}$ in $\operatorname{ker} A, X_{2}$ in a complement. Let $Q$ be the projection of $C^{M}$ onto Range $(A)$ and $K$ the inverse of $A$ as mapping from the complement of $\operatorname{ker} A$ into $\operatorname{Range}(A)$. Then the equation can be written as (for $\lambda$ small enough)

$$
\begin{gathered}
A(I-K Q T(\lambda))\left\{X_{2}-(I-K Q T(\lambda))^{-1} K Q T(\lambda) X_{1}\right. \\
\left.-(I-K Q T(\lambda))^{-1} K Q g(\lambda, X)\right\} \\
\oplus-(I-Q)\left\{T(\lambda)(I-K Q T(\lambda))^{-1} X_{1}+(I-T(\lambda) K Q)^{-1} g(\lambda, X)\right. \\
\left.+T(\lambda)\left[X_{2}-(I-K Q T(\lambda))^{-1} K Q\left(T(\lambda) X_{1}+g(\lambda, X)\right)\right]\right\}=0 .
\end{gathered}
$$

Now it is easily seen that the first part of the equation is uniquely solvable for $X_{2}=X_{2}\left(\lambda, X_{1}\right)$ for $\left(\lambda, X_{1}\right)$ small enough, with $\left\|X_{2}\right\| \leqslant C\left(\|\lambda\|\left\|X_{1}\right\|\right)+o\left(\left\|X_{1}\right\|\right)$. Thus the equation is equivalent to

$$
\tilde{B}(\lambda) X_{1}-\tilde{g}\left(\lambda, X_{1}\right)=0 \quad \text { on } \operatorname{ker} A
$$

with

$$
\begin{aligned}
& \tilde{B}(\lambda)=(I-Q) T(\lambda)(I-K Q T(\lambda))^{-1} \\
& \tilde{g}\left(\lambda, X_{1}\right)=-(I-Q)(I-T(\lambda) K Q)^{-1} g\left(\lambda, X_{1}+X_{2}\left(\lambda, X_{1}\right)\right),
\end{aligned}
$$

and

$$
\tilde{g}\left(\lambda, X_{1}\right)=o\left(\left\|X_{1}\right\|\right), \quad \tilde{B}(\lambda) \text { invertible for } \lambda \neq 0
$$

(since $B(\lambda)$ is invertible for $\lambda \neq 0$, if $B(\lambda) X=0$ then $\tilde{B}(\lambda) X_{1}=0$ ) and $\tilde{B}(0)=0$ (see [I1 and I2]). Note that the equivariance is preserved.

Furthermore, the following equivariant deformations are admissible for $\|\lambda\|=\rho$, $\|X\|=r$, and so give the same obstruction sets: (i) replace $g(\lambda, X)$ by $\operatorname{tg}(\lambda, X)$, $0 \leqslant t \leqslant 1$; (ii) replace $T(\lambda)$ by $T(t \lambda)$ in the first and in the last parts, so that the equation is deformed to $A X_{2} \oplus \tilde{B}(\lambda) X_{1}$. Let $E$ be an isomorphism of $\mathbf{C}^{M}$, which sends $(I-Q) C^{M}$ isomorphically onto $\operatorname{ker} A$ and $\mathscr{R}(A)$ into the complement of $\operatorname{ker} A$. Let $E(t)$ be a path of invertible matrices such that $E(0)=I, E(1)=E$. Then the deformation $E(t) A X_{2} \oplus E(t) \tilde{B}(\lambda) X_{1}$ is valid. After deforming $E A$ to $I$, one gets the matrix $X_{2} \oplus E \tilde{B}(\lambda) X_{1}$, which is a suspension and so has its primary obstruction zero, unless $k=2 s \leqslant 2 d$ : if $k$ is odd and $k \leqslant 2 M$ this was already proved, $B(\lambda)$ is deformable to $I$; if $2 d<k=2 s \leqslant 2 M$, then $B(\lambda)$ and $\left(I^{M-d}, E \tilde{B}(\lambda)\right)$ have the same (stable) class, but $\left(I^{s-d}, E \tilde{B}(\lambda)\right)$ is trivial since the image of $(1,0, \ldots, 0)^{T}$ under 
this matrix is constant (see $\S$ II.4.B); if $k>2 M$, the class of $B(\lambda) X_{0}$ is 0 for the same reason. Q.E.D.

However, by considering the equivalent (local) equation

$$
\tilde{B}(\lambda) X_{1}-\tilde{g}\left(\lambda, X_{1}\right)=0 \text { on } \operatorname{ker} A \cong \mathbf{C}^{d},
$$

one may apply the previous results for the case $k>2 d$ : a primary obstruction given by the class of $\tilde{B}(\lambda) X_{0}$ in $\Pi_{k-1}\left(S^{2 d-1}\right)$ and, if $k=2 d$, bifurcation in all directions. By applying the argument of global bifurcation to a neighborhood of $\left(X_{1}=0, \lambda=0\right)$, where this reduction is valid, one will get a continuum but the alternative may be false. In fact, the global index is zero if $k$ is odd or if $k$ is larger than $2 \max \left(\operatorname{dim} \operatorname{ker}\left(\operatorname{im}_{j} \beta I-L(\mu)\right)\right)$. Note that here $d \leqslant[M / 2]$.

II.4.7. Secondary obstructions. If $k>2 d$, then the previous obstruction was the first one only (for $k \leqslant 2 d$ it is the only one). If it vanishes, then $\tilde{B}(\lambda)$ is the double suspension of $C_{1}(\lambda)$, an element of $\mathrm{GL}\left(\mathbf{C}^{d-1}\right)$.

The next obstruction is in $H^{k+2}\left(K, L ; \Pi_{k-1}\left(S^{2 d-1}\right)\right)$ for $p=1$. Writing, as before, $X_{1}$ as $\tilde{X} \oplus X_{0}, \tilde{X}$ in $\mathbf{C}^{p}$, the second obstruction set is the same for the reduced equation (6) and for the section $\left(\lambda,\left[\tilde{X}, X_{0}\right]\right) \rightarrow\left(\lambda,\left[\tilde{X}, X_{0}, \tilde{X}, C_{1}(\lambda) X_{0}\right]\right)$. It contains the class of $(\tilde{X}, \lambda) \rightarrow\left(\tilde{X}, C_{1}(\lambda)(1,0, \ldots, 0)^{T}\right)$ in $\Pi_{k+1}\left(S^{2 d-1}\right)$, as before, that is $\Sigma^{2} P_{*} C_{1}(\lambda)$. Note that one may replace $C_{1}(\lambda)$ by $\delta(\alpha) C_{1}(\lambda)$, where $\alpha$ is any element of $\Pi_{k}\left(S^{2 d-1}\right)$ and $\delta$ is the coboundary operator in the exact sequence for the unitary group: then $i_{*} \delta(\alpha) C_{1}(\lambda)=B(\lambda)$, from the exactness. However, $\Sigma^{2} P_{*} C_{1}(\lambda)$ and $\Sigma^{2} P_{*} \delta(\alpha) C_{1}(\lambda)$ may be different and the fact that $\Sigma^{2} P_{*} C_{1}(\lambda)$ is nontrivial for some $C_{1}(\lambda)$ does not imply that $B(\lambda) X$ cannot be extended, as the following example shows: Let $d=3, k=7$ and consider the exact sequence

$$
\begin{aligned}
\Pi_{7}\left(U_{3}\right) & \rightarrow \Pi_{7}\left(S^{5}\right) \stackrel{\delta}{\rightarrow} \Pi_{6}\left(U_{2}\right) \stackrel{i}{\rightarrow} \Pi_{6}\left(U_{3}\right) \\
& \stackrel{P_{*}}{\rightarrow} \Pi_{6}\left(S^{5}\right) \stackrel{\delta_{1}}{\rightarrow} \Pi_{5}\left(U_{2}\right) \stackrel{i_{1}}{\rightarrow} \Pi_{5}\left(U_{3}\right) \stackrel{\tilde{P}_{*}}{\rightarrow} \Pi_{5}\left(S^{5}\right) .
\end{aligned}
$$

From Bott's theorem, $\Pi_{5}\left(U_{3}\right) \cong Z$ and $\tilde{P}_{*}$ sends the generator in a mapping of degree $(d-1) !=2$, hence $\tilde{P}_{*}$ is one-to-one. Also from Bott's theorem, $\Pi_{2 d}\left(U_{d}\right)$ is $Z_{d !}$ (or see [Bo]) so that $\Pi_{6}\left(U_{3}\right) \cong Z_{6}$. Now $U_{2}$ is isomorphic to $S^{3} \times S^{1}$ [St, p. 129] so that $\Pi_{5}\left(U_{2}\right) \cong Z_{2} \cong \Pi_{6}\left(S^{5}\right)$ and $\delta_{1}$, being onto, must be one-to-one, $P_{*}$ is 0 . Finally $\Pi_{7}\left(S^{5}\right)=Z_{2}[\mathrm{Hu}], \Pi_{7}\left(U_{3}\right)=0[\mathrm{Bo}] . \Pi_{6}\left(U_{2}\right) \cong \Pi_{6}\left(S^{3}\right) \cong Z_{12}[\mathrm{Hu}$, p. 329], where the first isomorphism is given by $P_{1}^{*}$ in the sequence for the product bundle

$$
U_{1} \rightarrow U_{2} \stackrel{P_{1}}{\rightarrow} S^{3}
$$

The situation is then

$$
\begin{aligned}
& 0 \quad \rightarrow \quad \Pi_{7}\left(S^{5}\right) \stackrel{\delta}{\rightarrow} \Pi_{6}\left(U_{2}\right) \stackrel{i}{\rightarrow} \Pi_{6}\left(U_{3}\right) \stackrel{P_{*}}{\rightarrow} \quad 0 \\
& 0 \rightarrow Z_{2} \quad \rightarrow \quad Z_{12} \quad \rightarrow \quad Z_{6} \quad \rightarrow \quad 0 \\
& \downarrow P_{1}^{*} \\
& \Pi_{6}\left(S^{3}\right) \stackrel{\Sigma}{\rightarrow} \Pi_{7}\left(S^{4}\right) \stackrel{\Sigma}{\rightarrow} \Pi_{8}\left(S^{5}\right) \\
& Z_{12} \quad \rightarrow \quad Z \oplus Z_{12} \quad \rightarrow \quad Z_{24}
\end{aligned}
$$


[Hu, pp. 329, 330]. Then, if $\gamma(\lambda)$ is the generator of $\Pi_{6}\left(U_{2}\right),\left({ }_{\gamma(\lambda)}^{I}\right)$ generates $\Pi_{6}\left(U_{3}\right), \gamma^{6}(\lambda)$ generates ker $i$ and the first obstruction for any element of $\Pi_{6}\left(U_{3}\right)$ always vanishes. Now $P_{1}^{*} \gamma$ generates $\Pi_{6}\left(S^{3}\right), \Sigma P_{1}^{*} \gamma$ generates the torsion component of $\Pi_{7}\left(S^{4}\right)$. The free component is generated by the Hopf map for quaternions $q$ and $\Sigma q$ generates $\Pi_{8}\left(S^{5}\right)$. Finally, $\Sigma^{2} P_{1}^{*} \gamma=2 \Sigma q$ (see the above reference for these results) implies that $\Sigma^{2}$ is one-to-one.

Now given any element of $\Pi_{6}\left(U_{3}\right)$, it can be written as

$$
\left(\begin{array}{ll}
I & \\
& \gamma^{s}
\end{array}\right), \quad s=0,1, \ldots, 5, \text { or }\left(\begin{array}{ll}
I & \\
& \gamma^{s+6}
\end{array}\right) .
$$

But $\Sigma^{2} P_{1}^{*}\left(\gamma^{s}\right)=2 s \Sigma q$ and $\Sigma^{2} P_{1}^{*}\left(\gamma^{s+6}\right)=(2 s+12) \Sigma q$, which are two different elements. Furthermore, $\left({ }^{I} \gamma^{6}\right)$ is deformable to the identity, and hence all its obstruction sets contain 0 , but $\Sigma^{2} P_{1}^{*}\left(\gamma^{6}\right)=12 \Sigma q \neq 0$. This shows that secondary obstructions may not be very useful for positive results (i.e. existence of bifurcation) unless the matrix $B(\lambda)$ has a very special form.

Assume now that $\Sigma^{2} P^{*} C_{1}(\lambda)=0$ and that one is in the stable range for the suspension, i.e. $k<4 d-6$. Hence $\Sigma^{2}$ is an isomorphism and $P^{*} C_{1}(\lambda)=0$, that is $C_{1}(\lambda)$ is deformable to $\left({ }^{I} C_{2}(\lambda)\right)$. The next obstruction, for extension on $\bar{K}^{k+4}$, will be the class of $\left(x_{1}, x_{2}, C_{2}(\lambda)(1,0, \ldots, 0)^{T}\right)=\Sigma^{4} P^{*} C_{2}(\lambda)$. If it vanishes and $\Sigma^{4}$ is an isomorphism, true for $k<4 d-10$, then $C_{2}(\lambda)$ is the suspension of $C_{3}(\lambda)$ with obstruction, for extension to $\bar{K}^{k+6}, \Sigma^{6} P^{*} C_{3}(\lambda)$, and so on. To go all the way to $\bar{K}^{k+2 d-2}$ would require $B(\lambda)$ deformable to $I$. It would be interesting to know under which circumstances the existence of an extension for $B(\lambda) X$ would imply the triviality of $B(\lambda)$ (true if $k \leqslant 2 d$ ). Note that if one had not reduced the problem to $\operatorname{ker} B(0)$ in Remark II.4.6, one would get one of the higher obstructions with less definite results. Note also that, if $k>2 d$, the stable class of $B(\lambda)$ is always zero.

II.5. The nonfree action case.

II.5.A. Fixed point subspaces of isotropy subgroups. Recall that the bifurcation equation

$(*)_{h}$

$$
B_{j}(\lambda) X_{j}-g_{j}(\lambda, X)=0, \quad j=1, \ldots, l,
$$

has the property that

$$
g_{j}\left(\lambda, e^{i \phi} X\right)=e^{i m, \phi} g_{j}(\lambda, X)
$$

where $e^{i \phi} X \equiv\left(e^{i \phi} X_{1}, e^{i m_{2} \phi} X_{2}, \ldots, e^{i m_{l} \phi} X_{l}\right)$. Let $M_{j_{0}}$ be the set of all $j$ 's in $\{1, \ldots, l\}$ such that $m_{j}$ is a multiple of $m_{j_{0}}$ (e.g. $\left.M_{1}=\{1, \ldots, l\}\right)$. Then if one takes $X_{j}=0$ for all $j$ 's in $M_{1}-M_{j_{0}}$, the corresponding $g_{j}(\lambda, X)$ are zero: in fact

$$
g_{j}\left(\lambda, e^{2 \pi i / m_{i_{0}}} X\right)=g_{j}(\lambda, X)=e^{2 \pi i m_{j} / m_{/ 0}} g_{j}(\lambda, X)
$$

from the choice of $X_{j}$ and the fact that $m_{j} / m_{0}$ is not an integer. This argument can also be used to eliminate all nonmultiples of $m_{j_{0}}$ in the Fourier series. Equivalently, this can be achieved by looking for solutions with $\nu$ close to $m_{j_{0}} \beta$, obtaining a stratification of the possible solutions. This fact has to be taken into account in order to construct an equivariant extension $[\mathbf{P a}, \mathbf{K o}]$ and requires a delicate analysis of these sets. However, one can reduce the nonfree case to a free action problem by considering the following arguments. 
II.5.B. Auxiliary problem. If $Z$ is a vector in $\mathbf{C}^{M}$, denote $Z^{s} \equiv\left(Z_{1}^{s}, \ldots, Z_{M}^{s}\right)$, for any positive integer $s$. Let $S^{2 / M-1} \equiv\left\{\left(\eta_{1}, \ldots, \eta_{l}\right), \eta_{j} \in C^{M}, \Sigma\left|\eta_{j}\right|^{2}=1\right\}$ be the unit sphere in $\mathbf{C}^{\prime M}$ and consider the system

$$
\left[B_{j}(\lambda) \tilde{r} \eta_{j}^{m_{j}}-g_{j}\left(\lambda, \tilde{r} \eta_{1}^{m_{1}}, \tilde{r} \eta_{2}^{m_{2}}, \ldots, \tilde{r} \eta_{l}^{m_{l}}\right)\right]^{\gamma_{j}}=0, \quad j=1, \ldots, l,
$$

with $\tilde{r}=r\left(\sum\left|\eta_{j}\right|^{2 m_{j}}\right)^{-1 / 2} \cdot \gamma_{j}$ stands for $m / m_{j}$, with $m=\prod_{i=1}^{l} m_{i}$. This notation will be used throughout $\S I I .5$.

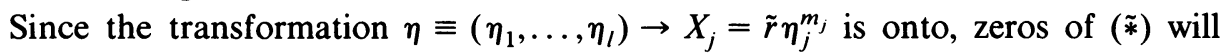
give zeros of $(*)_{b}$ and each zero of $(*)_{b}$ will give many roots for $(\tilde{*})$; extensions without zeros of $(*)_{b}$ to $B^{k} \times S^{2 / M-1}$ will give extensions without zeros of $(\tilde{*})$ but $n o t$ conversely. Thus, if the obstruction sets for $(\tilde{*})$ do not contain 0 , then $(*)_{b}$ will have a solution. (Note that this is also true if one does not take powers of the equations; this is done in order to compute the obstruction but one could apply the theory to that case too.)

Now if one has the standard action on $S^{2 l M-1}\left(e^{i \phi} \eta \equiv\left(e^{i \phi} \eta_{1}, \ldots, e^{i \phi} \eta_{l}\right)\right)$, then $(\tilde{*})\left(e^{i \phi} \eta\right)=e^{m \phi}(\tilde{*})(\eta)$. One gets a cross-section of the fiber bundle (the action on the total space is $\left.e^{i \phi}(\eta, Y)=\left(e^{i \phi} \eta, e^{i m \phi} Y\right)\right)$ and one is back to the previous situation.

The primary obstruction, being unique, is the same for $(\tilde{*})$ and for the system with $g_{j}$ deformed to 0 and $\tilde{r}$ to 1 . One may also deform $B_{j}(\lambda)$ to any other matrix in its class in $\Pi_{k-1}\left(\mathrm{GL}\left(\mathrm{C}^{M}\right)\right)$.

II.5.C. Case $k=2$. Since this case is easier it will be treated first. It has been seen that $B_{j}(\lambda)$ is deformable to the suspension of det $B_{j}(\lambda)$. Hence, the obstruction sets for $(\tilde{*})$ are those for

$$
\begin{gathered}
{\left[\left(\begin{array}{cc}
I & \\
& \operatorname{det} B_{1}(\lambda)
\end{array}\right) \eta_{1}\right]^{m}=\left(\begin{array}{ll}
I & \\
\vdots & \operatorname{det} B_{1}(\lambda)^{m}
\end{array}\right) \eta_{1}^{m},} \\
{\left[\left(\begin{array}{ll}
I & \operatorname{det} B_{l}(\lambda)
\end{array}\right) \eta_{l}^{m_{l}}\right]^{\gamma_{l}}=\left(\begin{array}{ll}
I & \operatorname{det} B_{l}(\lambda)^{\gamma_{l}}
\end{array}\right) \eta_{l}^{m},}
\end{gathered}
$$

since the matrices are diagonal and the determinant of a product is the product of the determinants. The above matrix, having the same determinant as the suspension of $\operatorname{det} \Pi B_{j}(\lambda)^{\gamma_{j}}$, may be equivariantly deformed to

$$
\left(\begin{array}{ll}
I & \\
& \operatorname{det} \prod B_{j}(\lambda)^{\gamma_{j}}
\end{array}\right) \eta^{m}
$$

(one may also use the direct deformation of [I1, p. 10]). The first obstruction (and the only one) is then the degree of the mapping $(\lambda, \tilde{\eta}) \rightarrow\left(\tilde{\eta}^{m}, \operatorname{det} \Pi B_{j}(\lambda)^{\gamma_{j}}\right)$ for $(\lambda, \tilde{\eta})$ on $\partial\left(B^{2} \times B^{2 / M-2}\right)$. Using the product theorem, this degree is $m^{M l-1} \sum$ Degree $B_{j}(\lambda)^{\gamma_{j}}$, that is

$$
\left(\prod m_{i}\right)^{M l} \sum_{n_{j}}(2) / m_{j} .
$$

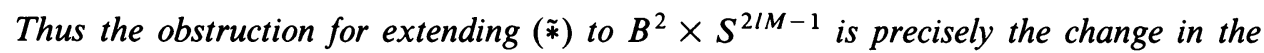
Fuller's degree given in [CMY]. Its vanishing implies the existence of an extension for (*) but not necessarily for $(*)_{b}$. 
II.5.D. Case $2<k \leqslant 2 M$. If $k$ is odd, each $B_{j}(\lambda)$ is deformable to $I$, giving a system which is extendible to $B^{k} \times S^{2 / M-1}$. Suppose then that $k$ is even, $k=2 s$, and deform $B_{j}(\lambda)$ to the suspension of $C_{j}(\lambda)$, an element of $\mathrm{GL}\left(C^{s}\right)$. One has to look at the map

$$
(\lambda, \eta) \rightarrow\left(\tilde{\eta}^{m},\left(C_{l}(\lambda) \xi_{l}^{m_{l}}\right)^{\gamma_{l}}, \ldots,\left(C_{1}(\lambda) \xi_{1}\right)^{m}\right)
$$

with $\xi_{j}$ in $\mathbf{C}^{s}$ and $\tilde{\eta}$ in $\mathbf{C}^{(M-s)}$. This map is equivariantly homotopic to

$$
\begin{aligned}
\left(\tilde{\eta}^{m},\left(C_{l}(\lambda) \xi_{l}^{m_{l}}\right)^{\gamma_{l}},\left(C_{l-1}(\lambda) \xi_{l-1}^{m_{l-1}}\right)^{\gamma_{l-1}}\right. & +t \xi_{l}^{m}, \ldots,\left(C_{2}(\lambda) \xi_{2}^{m_{2}}\right)^{\gamma_{2}} \\
& \left.+t \xi_{3}^{m},\left(C_{1}(\lambda) \xi_{1}^{m_{1}}\right)^{m}+t \xi_{2}^{m}\right), \quad \text { with } t \text { in }[0,1] .
\end{aligned}
$$

Now extending $C_{j}(\lambda)$ by $\rho^{-1}|\lambda| C_{j}(\lambda \rho /|\lambda|)$ and putting $t \equiv \rho^{2}-|\lambda|^{2}$, one gets a nonzero equivariant extension to $\bar{K}^{2 M l-1}$, i.e. for the points $(\lambda,[\eta])$, with $|\lambda| \leqslant \rho$, $\left[\tilde{\eta}, \xi_{1}, \ldots, \xi_{2}, 0\right]$. The first obstruction is then the degree of the above mapping from the boundary of the ball $|\lambda| \leqslant \rho,\left\|\tilde{\eta}, \xi_{1}, \ldots, \xi_{2}\right\| \leqslant 1$ into $\mathbf{C}^{M l}-\{0\}$, where $\xi_{1}$ has been replaced by $(1,0, \ldots, 0)^{T}$.

Consider now the map $\left(C(\lambda)(1,0, \ldots, 0)^{T}\right)^{m}=\left(P_{*} C(\lambda)\right)^{m}$ from $S^{2 s-1}=\{\lambda /|\lambda|$ $=\rho\}$ into itself: it is the composition of $P_{*} C(\lambda)$ with the application $\xi \rightarrow \xi^{m}$, which has degree $m^{s}$; this implies, from the composition theorem for degree, that $\operatorname{deg}\left(P_{*} C(\lambda)\right)^{m}=m^{s} \operatorname{deg} P_{*} C(\lambda)=\operatorname{deg} P_{*} C(\lambda)^{m^{s}}$, since $P_{*}$ is a morphism. Then $\left(P_{*} C(\lambda)\right)^{m}$ and $P_{*} C(\lambda)^{m^{s}}$, having the same degree, are homotopic one to the other by Pontryagin's theorem (see [Sp, p. 398]). If $G(\lambda, \tau)$ is the homotopy, then, replacing $\left(C_{1}(\lambda)(1,0, \ldots, 0)^{T}\right)^{m}+t \xi_{2}^{m}$ by $\rho^{-1}|\lambda| G(\lambda \rho /|\lambda|, \tau)+t \xi_{2}^{m}$, the obstruction is the degree of the map

$$
\left(\tilde{\eta}^{m},\left(C_{l}(\lambda) \xi_{l}^{m_{l}}\right)^{\gamma_{l}},\left(C_{l-1}(\lambda) \xi_{l-1}^{m_{l-1}}\right)^{\gamma_{l-1}}+t \xi_{l}^{m}, \ldots, C_{1}(\lambda)^{m^{s}}(1,0, \ldots, 0)^{T}+t \xi_{2}^{m}\right),
$$

which corresponds to an extension of the equivariant map on $S^{k-1} \times S^{2 / M-1}$ :

$$
(\lambda, \eta) \rightarrow\left(\tilde{\eta}^{m},\left(C_{l}(\lambda) \xi_{l}^{m_{l}}\right)^{\gamma_{1}}, \ldots,\left(C_{2}(\lambda) \xi_{2}^{m_{2}}\right)^{\gamma_{2}}, C_{1}(\lambda)^{m^{s}} \xi_{1}^{m}\right) .
$$

By replacing $\left(\xi_{2}, \xi_{1}\right)$ in this map by $\left(\tau \xi_{2}+(1-\tau) \xi_{1}, e^{i(1-\tau)}\left(\tau \xi_{1}-(1-\tau) \xi_{2}\right)\right)$ and, afterwards, $(A, B)$ by $\left(\tau A+(1-\tau) B, e^{i(1-\tau)}(\tau B-(1-\tau) A)\right)$, with $A=$ $\left(C_{2}(\lambda) \xi_{1}^{m_{2}}\right)^{\gamma_{2}}, B=C_{1}(\lambda)^{m^{s}} \xi_{2}^{m}$, one has an equivariant homotopy to the map

$$
\left(\tilde{\eta}^{m},\left(C_{l}(\lambda) \xi_{l}^{m_{l}}\right)^{\gamma_{l}}, \ldots, C_{1}(\lambda)^{m^{s}} \xi_{2}^{m},\left(C_{2}(\lambda) \xi_{1}^{m_{2}}\right)^{\gamma_{2}}\right) \text {. }
$$

The previous extension, Pontryagin's theorem and the fact that primary obstructions depend only on the equivariant homotopy class of maps on $S^{k-1} \times S^{2 / M-1}$ and are unique imply that the obstruction is the same for the initial map and for

$$
\left(\tilde{\eta}^{m},\left(C_{l}(\lambda) \xi_{l}^{m_{l}}\right)^{\gamma_{l}}, \ldots, C_{2}(\lambda)^{\gamma_{2}^{s}} \xi_{2}^{m}, C_{1}(\lambda)^{m^{s}} \xi_{1}^{m}\right)
$$

(after undoing the previous deformations). Repeating this process with $\left(\xi_{3}, \xi_{1}\right)$, $\left(\xi_{4}, \xi_{1}\right), \ldots,\left(\xi_{1}, \xi_{1}\right)$ one gets to the obstruction for the map

$$
\left(\tilde{\eta}^{m}, C_{l}(\lambda)^{\gamma_{l}^{i}} \xi_{l}^{m}, \ldots, C_{1}(\lambda)^{\gamma_{1}^{s}} \xi_{1}^{m}\right) .
$$

Now, if $A$ and $B$ belong to $\mathrm{GL}\left(\mathbf{C}^{M}\right)$, the following deformation is valid:

$$
\left(\begin{array}{cc}
1-\tau & \tau \\
-\tau & 1-\tau
\end{array}\right)\left(\begin{array}{cc}
(1-\tau) A & -\tau A B \\
\tau I & (1-\tau) B
\end{array}\right)
$$


since, if $\left(X_{1}, X_{2}\right)$ belongs to the kernel of this matrix, then

$$
X_{1}=-(1-\tau) \tau^{-1} B X_{2}, \quad\left((1-\tau)^{2} \tau^{-1}+\tau\right) A B X_{2}=0,
$$

so that $X_{2}=0=X_{1}$ (for $\tau=0$ this is clear). Applying this deformation to the above map, one has an equivariant homotopy to

$$
\left(\tilde{\eta}^{m}, \xi_{l}^{m}, \ldots, \xi_{2}^{m}, \prod_{j=1}^{l} C_{j}(\lambda)^{\gamma_{j}^{s}} \xi_{1}^{m}\right),
$$

where the product is of form $C_{l} C_{l-1} \cdots C_{1}$.

The obstruction is now the degree of the map

$$
\left(\tilde{\eta}, \xi_{l}, \ldots, \xi_{2}, \lambda\right) \rightarrow\left(\tilde{\eta}^{m}, \xi_{l}^{m}, \ldots, \xi_{2}^{m}, \prod C_{j}(\lambda)^{\gamma_{j}^{s}}(1,0, \ldots 0)^{T}\right),
$$

i.e. $m^{\prime M-s} \sum_{j} n_{j}(k)^{\gamma_{j}^{s}}(s-1)$ !, with $n_{j}(k)$ the class of $C_{j}(\lambda)$ in $\Pi_{k-1}\left(\mathrm{GL}\left(\mathbf{C}^{k / 2}\right)\right)$.

The change of the generalized Fuller's degree is then

$$
\left(\Pi m_{i}\right)^{l M}\left(\frac{k}{2}-1\right) ! \sum_{j} \frac{n_{j}(k)}{m_{j}^{k / 2}} .
$$

Note that one has not proved that the initial map is equivariantly homotopic to the last map (this was true for the case $k=2$ ), but only that these maps have the same primary obstruction. Thus, if the degree is zero, one may have higher obstructions or no extension for the initial map. The fact that it is enough to have $n_{j}(k) \neq 0$ for some $j$, will be proved in Remark II.5.3 below.

REMARKS. II.5.1. The unstable case. For $k>2 M$, the above extension to $\bar{K}^{2 M l+k-2 M-2}$ leads to an obstruction in $H^{k+2 p}\left(K, L ; \Pi_{k+2 p-1}\left(S^{2 M l-1}\right)\right)$, with $p=M(l-1)$. However, the first obstruction is for $p_{0}=M l-k / 2$ if $k$ is even and less or equal to $2 M l$; for $p_{0}=M l-(k-1) / 2$ if $k$ is odd and less or equal to $2 M l+1$; and for $p_{0}=0$ if $k>2 M l$. Since $p_{0}<p$ (except for $\left.k=2 M+1\right)$ the primary obstruction is zero and, as it has been seen in the last section, the nonvanishing of secondary obstruction does not imply positive results.

If $k=2 M+1$, the above arguments, appropriately modified, still work: in fact $\left(P_{*} B(\lambda)\right)^{m}$ is homotopic to $P_{*} C(\lambda)^{m^{M}}$ because $P_{*} C(\lambda)$, as an element of $\Pi_{2 M}\left(S^{2 M-1}\right)$, is either 0 if $M=1$ or a suspension if $M>1$; but then the right composition is associative, i.e. $\left(\alpha_{1}+\alpha_{2}\right) \circ \Sigma \beta=\alpha_{1} \circ \Sigma \beta+\alpha_{2} \circ \Sigma \beta$ if $\beta$ belongs to $\Pi_{p-1}\left(S^{r-1}\right)$ and $\alpha_{1}$ to $\Pi_{r}\left(S^{m}\right)$ (here $p=2 M, r=2 M-1=m$ ) [To, p. 16, 2.1]. The obstruction (primary) is then the class of $\left(\eta_{l}^{m}, \ldots, \eta_{2}^{m}, \Pi B_{j}(\lambda)^{\gamma_{j}}(1,0, \ldots, 0)^{T}\right)$ in $\Pi_{2 / M}\left(S^{2 / M-1}\right)=Z_{2}$. Since the mapping $\left(\eta_{l}, \ldots, \eta_{2}, \lambda\right) \rightarrow\left(\eta_{l}^{m}, \ldots, \eta_{2}^{m}, \lambda\right)$ has degree $m^{(l-1) M}$ and one has associativity for left composition $\left(\alpha \circ\left(\beta_{1}+\beta_{2}\right)=\alpha \circ \beta_{1}+\right.$ $\alpha \circ \beta_{2}$, see the above reference), the obstruction is

$$
\left(\prod m_{i}\right)^{(l-1) M} \sum_{j} \prod_{i \neq j} m_{i}^{M} P_{*} B_{j}(\lambda) \quad \text { in } Z_{2} .
$$

This class will be 0 if one of the $m_{i}$ 's is even and, if all are odd, it will be the class of $\sum \gamma_{j}^{M} P_{*} B_{j}(\lambda)$. However, here $P_{*} B_{j}(\lambda)=0$, since $B_{j}(\lambda)=i \nu m_{j} I-L(\mu)$ is always deformable to a suspension $\left(B_{j}(0) \neq 0\right.$; see Remark II.4.6). 
THEOREM II.5.1. For $k>2 M$, the primary obstruction, considered on $\mathbf{C}^{M}$, is always 0 .

II.5.2. Reduction to the kernel. As it has just been pointed out, one needs to look more closely into the structure of $B_{j}(\lambda)$. As in the last section, one may reduce (locally) the bifurcation equation to the kernels of $B_{j}(0)$ and obtain an equivalent system

$$
\left[\tilde{B}_{j}(\lambda) \tilde{r} \eta_{j}^{m_{j}}-\tilde{g}_{j}\left(\lambda, \tilde{r} \eta_{1}^{m_{1}}, \ldots, \tilde{r} \eta_{l}^{m_{l}}\right)\right]^{\gamma_{j}}=0, \quad j=1, \ldots, l,
$$

where $\tilde{B}_{j}(0)=0, \eta_{j} \in \mathbf{C}^{\tilde{d}_{i}}$ with $\tilde{d}_{j} \equiv \operatorname{dim} \operatorname{ker} B_{j}(0) \leqslant d_{j}$. If $\mathrm{k} \leqslant 2 \min \left(\tilde{d}_{j}\right)$, then the invariants for $\tilde{B}_{j}(\lambda)$ and for $B_{j}(\lambda)$ are the same but $n_{j}(k)=0$ for all $j$ 's for which $k>2 \tilde{d}_{j}$ (see Theorem II.4.2). It will be seen now that there is not much gain in considering $(* *)$ instead of $(\tilde{*})$ if $l>1$, except for those $j$ 's such that $k=2 \tilde{d}_{j}+1$.

THEOREM II.5.2. Let $\tilde{M}=\sum \tilde{d}_{j}, \tilde{d}=\max \tilde{d}_{j}$. If $l>1$, the primary obstruction for $(* *)$ is

(a) $\left(\Pi m_{i}\right)^{\tilde{M}}(s-1) ! \Sigma_{I}$ Degree $\tilde{B}_{j}(\lambda) / m_{j}^{s}$, in $Z$, if $k=2 s \leqslant 2 \tilde{d}$ and $I=\{j / k \leqslant$ $\left.2 \tilde{d}_{j}\right\}$

(b) $\left(\Pi m_{i}\right)^{\tilde{M}-s} \sum_{J} \Pi_{i \neq j} m_{i}^{s} P_{*}\left(\tilde{B}_{j}(\lambda)\right)$, in $Z_{2}$, if $k=2 s+1 \leqslant 2 \tilde{d}+1$ and $J=\{j / k$ $\left.=2 \tilde{d}_{j}+1\right\}$,

(c) 0 otherwise.

Proof. Note first that $2 \tilde{M} \leqslant M$, since the sum of the dimensions of

$$
\operatorname{ker}\left( \pm i m_{j} \beta I-L(0)\right)
$$

cannot be larger than the dimension of the space. Then the obstructions are in $H^{k+2 p}\left(K, L ; \Pi_{k+2 p-1}\left(S^{2 \tilde{M}-1}\right)\right)$. The primary obstruction is for $p_{0}=\tilde{M}-k / 2$ if $k$ is even and $k \leqslant 2 \tilde{M}$; for $p_{0}=\tilde{M}-(k-1) / 2$ if $k$ is odd and $k \leqslant 2 \tilde{M}+1$; and for $p_{0}=0$ if $k \geqslant 2 \tilde{M}$.

(a) If $k \geqslant 2 \tilde{M}$, the primary obstruction is the class of the map

$$
\lambda \in S^{k-1} \rightarrow\left(\left(\tilde{B}_{1}(\lambda)(1,0, \ldots, 0)^{T}\right)^{\gamma_{1}}, 0, \ldots, 0\right),
$$

which is linearly deformable to $(0,1,0, \ldots, 0)$ since $l>1$, and has a zero class.

(b) If $k<2 \tilde{M}$, let $\{\sigma(1), \sigma(2), \ldots, \sigma(l)\}$ be a permutation of the set $\{1, \ldots, l\}$, such that $\tilde{d}_{\sigma(1)} \geqslant \tilde{d}_{\sigma(2)} \geqslant \cdots \geqslant \tilde{d}_{\sigma(l)}$. Let

$$
(* *)_{\sigma(j)} \equiv\left(\tilde{B}_{\sigma(j)}(\lambda) \eta_{\sigma(j)}^{m_{\sigma(j)}}\right)^{\gamma_{\sigma(j)}} \text {. }
$$

Let $\zeta$ be the vector of the first $\tilde{M}-\tilde{d}$ components of $\eta \equiv\left(\eta_{1}, \ldots, \eta_{l}\right)$. Then the map

$$
\left(\begin{array}{c}
(* *)_{\sigma(1)} \\
\vdots \\
(* *)_{\sigma(l)}
\end{array}\right)+t\left(\begin{array}{c}
0 \\
\zeta^{m}
\end{array}\right), \quad \text { with } t=\rho^{2}-|\lambda|^{2},
$$

is a good extension to $B^{k} \times \mathbf{C} P^{\tilde{M}-\tilde{d}-1}$, since if it is zero for $\lambda \neq 0$, then $\eta_{\sigma(1)}=$ $0, \ldots, \zeta=0, \ldots, \eta_{\sigma(l)}=0$ by cascading, so that $\eta=0$ which is a contradiction and, if $\lambda=0$, then $\zeta=0$. The next obstruction is then for $(\lambda, \zeta)$ in $B^{k} \times B^{2(\tilde{M}-\tilde{d})}$, i.e. for 
$p=\tilde{M}-\tilde{d}$. But then if $k>2 \tilde{d}$, for $k$ even, or if $k>2 \tilde{d}+1$, for $k$ odd, $p>p_{0}$ and thus the primary obstruction is zero.

(c) If $k=2 \tilde{d}+1$ and $\tilde{d}_{\sigma(l)}=\cdots=\tilde{d}_{\sigma(1)}$, the argument in Remark II.5.1 implies that the obstruction is $m^{(l-1) \tilde{d}} \sum_{j} \gamma_{j}^{\tilde{d}} P_{*} \tilde{B}_{j}(\lambda)$ in $Z_{2}$. While if $\tilde{d}_{\sigma(l)}<\tilde{d}$, the obstruction is the class of the map (10), with $\eta$ replaced by $\left(\zeta,\left(1-|\zeta|^{2}\right)^{1 / 2}, 0, \ldots, 0\right)$, for which $(* *)_{\sigma(l)}=0$. But then this is also the class for this map, where $\tilde{B}_{\sigma(l)}$ has been replaced by $|\lambda| I$ (or equivariantly by $I$ ), for which one could have left $\eta_{\sigma(l)}^{m}$ out of the extension. Then, if $\tilde{l}$ is the largest integer such that $\tilde{d}_{\sigma(j)}=\tilde{d}$ for $j \leqslant \tilde{l}$, by repeating the argument and using the fact that one is dealing with primary obstruction, one has to look at the obstruction for the map

$$
(* *)_{\sigma(j)}, \quad j \leqslant \tilde{l}, \quad \eta_{\sigma(j)}^{m}, \quad j \geqslant \tilde{l} .
$$

Thus, the class is

$$
m^{\tilde{M}-\tilde{l} \tilde{d}} m^{(\tilde{l}-1) \tilde{d}} \sum_{j \leqslant \tilde{l}} m^{\gamma_{\sigma(j)}^{\tilde{d}} P_{*} \tilde{B}_{\sigma(j)}}(\lambda)
$$

i.e. the same result as before, but where only the elements of $J$ are counted.

(d) If $k=2 s \leqslant 2 \tilde{d}$, let $l_{1}$ be the largest integer such that $2 \tilde{d}_{\sigma\left(l_{1}\right)} \geqslant k$. Then one may deform $B_{\sigma(j)}$ to the suspension of $C_{\sigma(j)}$, an $s \times s$ matrix, for $j \leqslant l_{1}$. If $(\tilde{*} \tilde{*})_{\sigma(j)}$ is the corresponding expression for $C_{\sigma(j)}$, let $\xi_{\sigma(j)}$ be in $\mathbf{C}^{s}$ and $\tilde{\eta}_{\sigma(j)}$ be in $\mathbf{C}^{\tilde{d}_{\sigma(j)}-s}$. Let $\zeta$ be the first $\sum_{j>l_{1}} \tilde{d}_{\sigma(j)}+\left(l_{1}-1\right) s$ coordinates of $\left(\xi_{\sigma(1)}, \ldots, \xi_{\sigma\left(l_{1}\right)}, \eta_{\sigma\left(l_{1}+1\right)}, \ldots, \eta_{\sigma(l)}\right)$. Then $\left(\tilde{\eta}^{m},(\tilde{*} \tilde{*})_{\sigma(1)}, \ldots,(\tilde{*} \tilde{*})_{\sigma\left(l_{1}\right)},(* *)_{\sigma\left(l_{1}+1\right)}, \ldots,(* *)_{\sigma(l)}\right)+\left(0,0,\left(\rho^{2}-|\lambda|^{2}\right) \zeta^{m}\right)$ is a valid extension to $\bar{K}^{\tilde{M}-s}$. As in the previous case, the matrices $B_{\sigma(j)}$, for $j>l_{1}$, can be replaced by the identity in the computation of the first obstruction. This is then the degree of $\left(\tilde{\eta}^{m}, \zeta^{m}, \Pi_{j \leqslant l_{1}} C_{\sigma(j)}^{\gamma_{\sigma(j)}^{s}}(1,0, \ldots, 0)^{T}\right)$; that is

$$
\left(\prod m_{i}\right)^{\tilde{M}}(s-1) ! \sum_{j \in I} \text { Degree } B_{j}(\lambda) / m^{k / 2} \text {. }
$$

(e) If $k=2 s+1<2 \tilde{d}$, by Bott's theorem, one may deform $B_{\sigma(j)}$ to $I$ for all $j$ 's with $2 \tilde{d}_{\sigma(j)}>k$ and, by the above argument, for all $j$ 's with $2 \tilde{d}_{\sigma(j)}+1<k$. One is left with the $j$ 's with $2 \tilde{d}_{o(j)}+1=k$. If $J$ is their set, with cardinality $\tilde{l}$, the primary obstruction is then the class, in $Z_{2}$, of $\left(\Pi m_{i}\right)^{\tilde{M}-s} \sum_{J} \Pi_{i \neq j} m_{i}^{s} P_{*} B_{j}(\lambda)$. Q.E.D.

II.5.3. From the considerations at the beginning of this section (reduction from the set $\{1, \ldots, l\}$ to $\left.M_{j_{0}}\right)$ and from the fact that $2 \pi / m_{j} \beta$ periodic solutions are also $2 \pi / \beta$ periodic, one may reduce the study of the nonfree case to the situation where $P_{*} B_{j}(\lambda)=0, j=2, \ldots, l$, and $P_{*} B_{1}(\lambda) \neq 0$. If $l=1$ one is back to the free case; if $2 \leqslant k \leqslant 2 M$, then $P_{*} B_{1}(\lambda)$ is the unique obstruction for extension of $(*)_{b}$, as it can be easily seen from the proof, and the above remarks may be adapted to this situation. However the above situation does not guarantee that the minimal period will be $2 \pi / \beta$ or, in the more general setting, that $X_{1}$ will be nonzero. In fact the system

$$
\lambda X_{1}-\left|X_{1}\right|^{2} X_{1}=0, \quad|\lambda|^{2} X_{2}-\left|X_{1}\right|^{2} X_{1}^{2}\left(1+\left|X_{2}\right|^{2}\right)=0,
$$

with $X_{1}, X_{2}, \lambda$ in $\mathbf{C}$, is equivariant under the action $e^{i \phi}\left(X_{1}, X_{2}\right)=\left(e^{i \phi} X_{1}, e^{2 i \phi} X_{2}\right)$. If $X_{1} \neq 0$, then $\lambda=\left|X_{1}\right|^{2},\left|X_{2}\right|=1+\left|X_{2}\right|^{2}$, leading to a contradiction. So $X_{1}=0$, $\lambda=0, X_{2}$ are the bifurcation solutions. 
III. Global bifurcation. In this part of the paper one will study the equation

$$
\nu \dot{X}=f(\mu, X)
$$

with $f(\mu, X) C^{1}$ in $X, f$ and $f_{X}$ Lipschitz in $\mu . X$ is in the space of $2 \pi$-periodic continuous functions $C^{0}\left(S^{1}\right)^{M}$, with $\mu$ in $\mathbf{R}^{k-1}$ and the period $T \equiv 2 \pi / \nu$ in $\mathbf{R}^{+}$.

Let $S$ be the set of stationary solutions $\{(X, \mu, T)$ with $f(\mu, X)=0$, any $T \geqslant 0\}$ and $P$ the set of nontrivial periodic solutions, i.e. $\left\{(X, \mu, T)\right.$ solution of $(*)_{\nu}, X(\tau)$ nonconstant $\}$.

It is easy to see that $\bar{P}-P \subset S[$ AY, I1, p. 124] and that these points are bifurcation points. If $\left(X_{p}, \mu_{p}, T_{p}\right)$ is such a point, then Lasota and Yorke have proved that $T_{p}>0$.

If $L_{p}\left(\mu_{p}\right) \equiv D_{X} f\left(\mu_{p}, X_{p}\right)$ is invertible, then there are an integer $q(p)$ and a positive number $\beta(p)$ such that $T_{p}=2 \pi q(p) / \beta(p)$ and $i \beta(p)$ belongs to the spectrum of $L_{p}\left(\mu_{p}\right)$ [AY, I2, p. 1248; MY, Appendix]. Furthermore, the stationary points near $\left(X_{p}, \mu_{p}\right)$ are of the form $X_{p}(\mu)$ and

$$
f(\mu, X)=L_{p}(\mu)\left(X-X_{p}(\mu)\right)+g_{p}(\mu, X)
$$

with $g_{p}(\mu, X)=o\left(\left\|X-X_{p}(\mu)\right\|\right)$.

Let $\nu_{p} \equiv 2 \pi / T_{p}$ and consider all eigenvalues of $L_{p}\left(\mu_{p}\right)$ of the form $m_{j}(p) \nu_{p}$, with $m_{1}(p)<m_{2}(p)<\cdots<m_{l_{p}}(p)$ integers. Note that it is not necessary that $m_{1}(p)$ $=1$, i.e. $\nu_{p}$ is not necessarily an eigenvalue of $L_{p}\left(\mu_{p}\right)$. For instance, for a linear problem with eigenvalues $\pm i, \pm 3 i / 2$ the solution $X e^{i t}+Y e^{3 i t / 2}$, with least period $4 \pi$, may be thought of as bifurcating from $\nu=1 / 2$, in which case $m_{1}=2, m_{2}=3$.

If $L_{p}(\mu)$ satisfies hypotheses H.1-H.3 for these eigenvalues, then one may apply the local bifurcation result.

Let $\mathscr{C}$ be the connected component of $\bar{P}$ branching off $(0,0,2 \pi / \beta)$. Assume $\mathscr{C}$ is bounded and that all bifurcation points in $\mathscr{C},\left(X_{p}, \mu_{p}, T_{p}\right)$, are such that the linearization $L_{p}(\mu)$ satisfies H.1, H.2 and H.3 (i.e. the real part of the corresponding eigenvalues of $L_{p}(\mu)$, for $\mu \neq \mu_{p}$, are nonzero). Since the intersections of $\bar{P}$ and $S$ with any bounded set are compact (this is true for the initial points $X(0)$ [I2, p. 1328] and then using the continuous dependence of the solution with respect to the initial conditions), then $\mathscr{C}$ is compact and there is only a finite number of bifurcation points on it ( $p \in I$ a finite set). Note that for each of these points, the local obstruction theory of $\S$ II may be applied.

III.1. Construction of an invariant neighborhood and reduction to finite dimension.

III.1.A. Construction of the neighborhood. As in the previous articles, one will construct a neighborhood of $\mathscr{C}$ such that $(*)_{\nu}$, has no solution on its boundary except for $(X, \mu, T)$ close to a bifurcation point. In fact one wants an open bounded set $\Omega$ in $C^{0}\left(S^{1}\right)^{M} \times \mathbf{R}^{k}$ with the following properties:

(1) $\mathscr{C} \subset \Omega$ and there are positive constants, $c, C$, such that $0<c \leqslant \nu \leqslant C$ on $\bar{\Omega}$.

(2) The only stationary points in $\bar{\Omega}$ are of the form $\left(X_{p}(\mu), \mu, T\right)$, with $(\mu, T)$ close to $\left(\mu_{p}, T_{p}\right), p$ in $I$. Furthermore, if $X(\tau)=X_{0}+Y(\tau), X_{0} \in \mathbf{R}^{M}, Y(\tau) L^{2}-$ orthogonal to the constants, then $\bar{\Omega}$ in a neighborhood of $\left(X_{p}, \mu_{p}, T_{p}\right)$ has the form of a ball $\|Y\| \leqslant \varepsilon_{2},\left\|X_{0}-X_{p}\right\| \leqslant \varepsilon_{0},\left\|\mu-\mu_{p}\right\|+\left|T-T_{p}\right| \leqslant \varepsilon_{k}$, where the local reduction done in the first part is valid, in particular where the only solution, with 
$\left\|\mu-\mu_{p}\right\|+\left|T-T_{p}\right|=\varepsilon_{k}$, is $Y=0, X_{0}=X_{p}(\mu)$. Finally, if $d((X, \mu, T) ; S) \equiv$ distance of $(X, \mu, T)$ in $\bar{\Omega}$ to $S$ (recall that $T$ does not play any role in $S$ ) is less than $\min \left(\varepsilon_{0}, \varepsilon_{1}\right)$, then $(X, \mu, T)$ is in one of the above balls. If $(0,0,2 \pi / \beta)$ is the only bifurcation point in $\mathscr{C}$, this distance function will be replaced by $\left\|X_{0}\right\|+\|Y\|$.

(3) If $X(\tau)$ is in $\Omega$, so is $X(\tau+\phi) \equiv X_{\phi}(\tau)$.

(4) $(*)_{\nu}$ has no solutions on $\partial \Omega$ except points of the form $\left(X_{p}(\mu), \mu, T\right)$, for $(\mu, T)$ close to $\left(\mu_{p}, T_{p}\right), p$ in $I$.

The construction of a neighborhood of $\mathscr{C}$ satisfying properties (1) and (2) is easy since $\mathscr{C}$ and $S$, intersected with a bounded set, are compact; the distance function is continuous and $f(\mu, 0)=0$. Now, since $\mathscr{C}$ is a connected component, if $(X, \mu, T)$ is in $\mathscr{C}$, so is $\left(X_{\phi}, \mu, T\right): \mathscr{C}$ is $S^{1}$ invariant. One may then start from any finite covering of $\mathscr{C}$ by balls satisfying (1) and (2) ( $\mathscr{C}$ is compact), replace these balls by their translate under the action, obtaining thus "tori" with the same properties (the action is an isometry for the max-norm, $S, \bar{P}$ and $\mathscr{C}$ being $S^{1}$ invariant) and a first invariant neighborhood. An inspection of the proof of "Whyburn's lemma"4 (see [14, Chapter III, §II]) shows that all the sets can be taken invariant (again using the isometry), getting thus an invariant $\Omega$ with $\partial \Omega \cap \bar{P}=\varnothing$.

III.1.B. Reduction to finite dimension. In this subsection one will use the compactness of the intergral operator, associated to $(*)_{\nu}$, in order to replace $(*)_{\nu}$ by an approximate finite-dimensional system $(\dagger)_{n}$. It is important to note that the approximation will not be needed in the rest of the argument, except for the fact that the two systems have the same obstruction sets in a vicinity of a bifurcation point. Thus, with this reduction, one avoids the construction of an obstruction theory for infinite-dimensional spaces, although such an extension could easily be done along the usual lines for degree theories.

Consider the equivalent equation

$$
\nu\left(X-X_{0}\right)-K f(\mu, X)=0, \quad X_{0} \equiv 2 \pi^{-1} \int_{0}^{2 \pi} X(\tau) d \tau .
$$

Here $K$ is the compact operator resulting from the composition

$$
C^{0}\left(S^{1}\right)^{M} \rightarrow L^{2}\left(S^{1}\right)^{M} \stackrel{\tilde{K}}{\rightarrow} W^{1.2}\left(S^{1}\right)^{M} \rightarrow C^{0}\left(S^{1}\right)^{M},
$$

where the first and last operators are inclusions, the last is compact from the Sobolev embedding theorem, and

$$
\tilde{K}\left(Y_{0}+\sum_{n \neq 0} Y_{n} e^{i n \tau}\right)=Y_{0}+\sum_{n \neq 0} Y_{n} \frac{e^{i n \tau}}{i n} .
$$

Since the Nemitsky operator associated to $f$ is Lipschitz continuous in $C^{0}\left(S^{1}\right)^{M}$ and $\tilde{K}$ is bounded, the resulting nonlinear operator is compact. The equation (12) is then the system $(*)_{n}$.

Since $K$ is compact and the equation (12) is nonzero on $A \equiv \partial \Omega \cap$ $\left\{(X, \mu, T) / d(X, \mu, T ; S) \geqslant \min \left(\varepsilon_{0}, \varepsilon_{1}\right)\right\}$, it is a standard argument that there is a positive $\varepsilon$ such that, on this set, $\left|X-X_{0}-\nu^{-1} K f(\mu, X)\right|_{C^{0}} \geqslant \varepsilon$.

\footnotetext{
${ }^{4}$ In fact this result goes back to the early days of topology, see [Ku, Chapter V].
} 
Let $P_{N}\left(\sum Y_{n} e^{i n \tau}\right)=\sum_{-N}^{N} Y_{n} e^{i n \tau}$ be the orthogonal projection in $W^{\varepsilon, 2}\left(S^{1}\right)^{M}, \varepsilon=0$, 1 , on the $2 N+1$ first modes $\left(Y_{-n}=\bar{Y}_{n}\right)$.

Let $Q_{N}=\sum_{0}^{N} P_{n} /(N+1)$ be the Cesàro sum. Since

$$
\left(Q_{N} X\right)(\tau)=2 \pi^{-1} \int_{-\pi}^{\pi} X(\tau+\eta) F_{N}(\eta) d \eta
$$

with $F_{N}(\eta)=(\sin (N+1) \eta / 2)^{2} /(N+1)(\sin \eta / 2)^{2}$, the Fejér kernel, it follows from Fejér's theorem that $Q_{N} X$ converges in $C^{0}\left(S^{1}\right)^{M}$ to $X$ and that $\left\|Q_{N}\right\|_{C^{0}} \leqslant 1$. Then there is an $N$ such that

$$
\left\|\nu^{-1}\left(I-Q_{n}\right) K f(\mu, X)\right\|_{C^{0}} \leqslant \varepsilon / 2 \text { for } n \geqslant N
$$

and for $(X, \mu, T)$ in the above set $A$ : if not, one has a sequence of $n_{j}, X_{j}, \mu_{j}, T_{j}$, with $n_{j}$ going to $\infty,\left(\mu_{j}, T_{j}\right)$ converging to $(\mu, T)$ (the set $A$ is closed), $K f\left(\mu_{j}, X_{j}\right)$ converging to $Y$ (for a subsequence by the compactness) and with the inequality reversed. But then $\left(I-Q_{n_{j}}\right)\left(K f\left(\mu_{j}, X_{j}\right)-Y\right)+\left(I-Q_{n_{j}}\right) Y$ tends to 0 , since $Q_{n_{,}}$is uniformly bounded and from above.

From both inequalities if follows that $\nu\left(X-X_{0}\right)-Q_{N} K f(\mu, X)$ is nonzero on the set $A$ for $N$ large enough. In particular, for elements of $Q_{N} C^{0}\left(S^{1}\right)^{M}$, i.e. for $Q_{N} X=\sum_{-N}^{N} X_{n} e^{i n \tau}(N+1-|n|) /(N+1)$, with $X_{-n}=\bar{X}_{n}$, in which case the equation $\nu\left(X-X_{0}\right)-Q_{N} K f(\mu, X)=0$ is equivalent to

$$
\operatorname{in} \nu X_{n}-f_{n}\left(\mu, Q_{N} X\right)=0, \quad 0 \leqslant n \leqslant N
$$

(since for negative $n$ 's one has the conjugates). Now, if $\left(Q_{N} X, \mu, T\right)$ belongs to $\partial \Omega_{N} \equiv Q_{N} C^{0}\left(S^{1}\right)^{M} \times \mathbf{R}^{k} \cap \partial \Omega$ and $d\left(Q_{N} X, \mu, T ; S\right)<\min \left(\varepsilon_{0}, \varepsilon_{1}\right)$, then, from the properties of $\Omega$, one has $\left|\mu-\mu_{p}\right|+\left|T-T_{p}\right|=\varepsilon_{k},\left\|X_{0}-X_{p}\right\| \leqslant \varepsilon_{0}$, $\left\|\sum_{0<|n| \leqslant N} \prod_{n \neq 0} X_{n} e^{i n \tau}(1-|n| /(N+1))\right\| \leqslant \varepsilon_{1}$, for some $p$ in $I$. There the equation $(\dagger)_{n}$ has the form

$$
\begin{gathered}
\left(\text { in } \nu I-L_{p}(\mu)(1-n /(N+1))\right) X_{n}-g_{p, n}\left(\mu, Q_{N} X\right)=0, \quad 0<n \leqslant N, \\
L_{p}(\mu)\left(X_{0}-X_{p}(\mu)\right)+g_{p, 0}\left(\mu, Q_{n} X\right)=0
\end{gathered}
$$

with $g_{p, n}\left(\mu, Q_{N} X\right)=o\left(\left\|Q_{N} X-X_{p}(\mu)\right\|\right)$. If $L_{p}(\mu)$ has $i \gamma$ as an eigenvalue, for $\mu$ close to $\mu_{p}$, and, if $\nu$ is close to $\beta(p) / q(p)$, then the above matrices will not be invertible if $\nu / \gamma=1 / n-1 /(N+1)$. Since $\gamma$ is bounded away from zero and bounded in that neighborhood, one has a bound on the possible $n$ 's (independent of $N$ ). By choosing $N$ very large, $\gamma$ has to be close to a multiple of $\nu$, that is close to a multiple of $\beta(p) / q(p)=\nu_{p}$. Thus $\gamma=m_{j}(p) \nu_{p}, n=m_{j}(p), \mu=\mu_{p}$. The above matrices are singular for $\nu_{p}-\nu=m_{j} \nu_{p} /(N+1)$ and invertible for $\left|\mu-\mu_{p}\right|+$ $\left|T-T_{\mu}\right|=\varepsilon_{k}$.

Now, since the spectrum of $L_{p}(\mu)$ is bounded and $|1-n /(N+1)| \leqslant 1$, there is an $N_{0}$ such that the inverses of the above matrices are bounded, from the spectral mapping, by $C / n$ for all $n \geqslant N_{0} ; N_{0}, C$ independent of $N$. For the other inverses (for $(\mu, \nu)$ on the boundary of the disc) there is an $N_{1}$ such that one has a similar bound if $N \geqslant N_{1}$. One gets then

$$
\begin{gathered}
\left|X_{n}\right| \leqslant \operatorname{Co}\left(\left|Q_{n} X-X_{p}(\mu)\right|\right) / n, \quad n>0, \\
\left|X_{0}-X_{p}(\mu)\right| \leqslant \operatorname{Co}\left(\left|Q_{N} X-X_{p}(\mu)\right|\right),
\end{gathered}
$$


so that

$$
\left\|Q_{N} X-X_{p}(\mu)\right\|_{C^{0}} \leqslant a\left\|Q_{N} X-X_{p}(\mu)\right\|_{W^{1,2}} \leqslant o\left(\left|Q_{N} X-X_{p}(\mu)\right|\right)
$$

( $a$ is given by Sobolev embedding), for all $N \geqslant N_{1}$. This implies that one may choose $\varepsilon_{0}, \varepsilon_{1}$ beforehand, so that any solution of $(\dagger)_{n}$, with $\left|\mu-\mu_{p}\right|+\left|T-T_{p}\right|=\varepsilon_{k}$ and $d\left(Q_{N} X, \mu, T ; S\right) \leqslant \min \left(\varepsilon_{0}, \varepsilon_{1}\right)$, must be $Q_{N} X=X_{p}(\mu)$ for any $N \geqslant N_{1}$.

Thus the pair $\left((\dagger)_{n}, 0 \leqslant n \leqslant N, d\left(Q_{N} X, \mu, T ; S\right)-\varepsilon\right)$ is nonzero, for any positive $\varepsilon$, for $\left(Q_{N} X, \mu, T\right)$ in $\partial \Omega_{N}$.

From now on $Q_{N} C^{0}\left(S^{1}\right)^{M}$ will be identified with $\mathbf{R}^{M} \times \mathbf{C}^{M N}$, by looking at the components $\left(X_{0}, X_{1}, \ldots, X_{N}\right) \equiv\left(X_{0}, Y\right)$, the maximum norm will be used and the image of $\Omega_{N}$ will be also called $\Omega_{N}$. On $\mathbf{C}^{M N}$ one has the action $e^{i \phi} Y \equiv$ $\left(e^{i \phi} X_{1}, e^{2 i \phi} X_{2}, \ldots, e^{N i \phi} X_{N}\right)$, which is an isometry under the maximum norm.

On the bounded, open, invariant set $\Omega_{N}$ one will consider the triple

$$
F_{\varepsilon}\left(X_{0}, Y, \mu, \nu\right) \equiv\left\{\begin{array}{l}
\text { in } \nu X_{n}-f_{n}\left(X_{0}, Y, \mu\right), \quad 0<n \leqslant N, \\
f_{0}\left(X_{0}, Y, \mu\right), \\
d\left(X_{0}, Y, \mu, \nu ; S\right)-\varepsilon,
\end{array}\right.
$$

with $f_{n}\left(X_{0}, Y, \mu\right) \equiv f_{n}\left(Q_{N} X, \mu\right)$, and $S$ is taken to be in $\mathbf{R}^{M} \times \mathbf{R}^{k}$, given by points of the form $\left(X_{0}, \mu, \nu\right)$. Note that $d\left(X_{0}, Y, \mu, \nu ; S\right)$ can be replaced by any continuous function on $\Omega_{N}$ with the property that if it vanishes on $\partial \Omega_{N}$, then $\left(X_{0}, \mu, \nu\right)$ belongs to $S$, for example by $\left\|X_{0}\right\|+\|Y\|$, if $\mathscr{C}$ contains only $(0,0,2 \pi / \beta)$ as a bifurcation point.

Then $F_{\varepsilon}$ is nonzero on $\partial \Omega_{N}$.

REMARK III.1.1. The operator $Q_{N}$, which is not a projection, is needed because of the lack of uniform convergence of the Fourier series for continuous functions. However, one could have worked with Fourier series in $W^{1,2}\left(S^{1}\right)$ from the start; in fact, from the regularity of solutions, $S$ and $P$ are contained in $W^{1.2} . \bar{P}-P \subset S$ is also valid, so that the closure of $P$ with respect to the $W^{1,2}$ norm is the closure of $P$ with respect to the uniform norm. Chains, in $C^{0}$ and in $W^{1,2}$, of solutions are identical, so that connected components of $\bar{P}$ are the same in both norms. One may then construct the set $\Omega$ in $W^{1,2}$, with the same properties. $f(\mu, X(\tau))$ is clearly a Lipschitz continuous operator on $C^{0}\left(S^{1}\right)^{M}$ and, since $W^{1,2}$ is compactly included in $C^{0}\left(S^{1}\right)$, the operator $K f$ is compact on $W^{1,2}\left(S^{1}\right)^{M}$. By considering the equation $\nu\left(X-X_{0}\right)-K f(\mu, X)$ on $W^{1,2}$, one may reduce the problem to finite dimension by using $P_{N}$ instead of $Q_{N}$. One gets locally the matrices inI $-L_{p}(\mu)$, which are clearly deformable to the previous ones for $\left|\mu-\mu_{p}\right|+\left|T-T_{p}\right|=\varepsilon_{k}$. Thus one has the same obstruction sets.

III.2. $S^{1}$-inessential mappings.

The next step in the proof is to replace the notion of obstruction by the weaker, but related, notion of equivariant essentiality.

Definition. Let $X$ be a closed, bounded, invariant subset of $\mathbf{C}^{M N} \times \mathbf{R}^{M} \times \mathbf{R}^{k}$. Let $F\left(Y_{0}, X_{0}, \lambda\right) \equiv\left(F_{1}, \ldots, F_{N}, F_{0}\right)$ be a mapping from $X$ into $\mathbf{C}^{M N} \times \mathbf{R}^{M+1}-\{0\}$, which is equivariant, i.e. $F_{j}\left(e^{i \phi}\left(Y_{0}, X_{0}, \lambda\right)\right)=e^{i j \phi} F_{j}\left(Y_{0}, X_{0}, \lambda\right), j=0, \ldots, N\left(F_{j}\right.$ belongs to $\mathbf{C}^{M}$ if $j \geqslant 1$ and to $\mathbf{R}^{M+1}$ if $j=0$, the action is the one defined above). 
Then, if $Y$ is a closed, bounded, invariant subset containing $X, F$ is called $S^{1}$-inessential with respect to $Y$ if and only if there is a nonzero equivariant extension of $F$ to $Y$.

Lemma. (1) If $F_{0}$ and $F_{1}$ are equivariantly homotopic on $X$ and $F_{0}$ is $S^{1}$-inessential with respect to $Y$, then so is $F_{1}$.

(2) $F$ is $S^{1}$-inessential with respect to any $Y$ (or with respect to a ball $B$, centered at the origin) if and only if $F$ is equivariantly deformable to $(0, \ldots, 0, c), c$ a constant in $\mathbf{R}^{M+1}$.

Proof. It is enough to adapt the standard proof to the equivariant case [14]. However, for sake of completeness, a quick review of the main points will be given here.

Let $\tilde{F}_{0}$ be the equivariant extension of $F_{0}$ and $F(X, t)$ be the equivariant homotopy from $F_{0}$ to $F_{1}\left(X\right.$ stands for $\left.\left(Y_{0}, X_{0}, \lambda\right)\right)$. Set $Z \equiv X \times[0,1] \cup Y \times\{0\}$ and $\tilde{F}$ : $Z \rightarrow \mathbf{C}^{M N} \times \mathbf{R}^{M+1}-\{0\}$ by

$$
\begin{array}{ll}
\tilde{F}(X, t)=F(X, t) & \text { if } X \in X, \\
\tilde{F}(Y, 0)=\tilde{F}_{0}(Y) & \text { if } Y \in Y .
\end{array}
$$

From Tietze's extension theorem, $\tilde{F}$ extends to $G^{1} \equiv\left(G_{1}^{1}, \ldots, G_{N}^{1}, G_{0}^{1}\right)$ from $Y$ into $\mathbf{C}^{M N} \times \mathbf{R}^{M+1}$. Define

$$
G_{j}(X, t)=(2 \pi)^{-1} \int_{0}^{2 \pi} e^{-i j \phi} G_{j}^{1}\left(e^{i \phi} X, t\right) d \phi, \quad j=0, \ldots, N .
$$

It is easy to see that $G$ is an extension of $\tilde{F}$ and that $G_{j}\left(e^{i \phi} X, t\right)=e^{i j \phi} G_{j}(X, t)$, by changing variables and periodicity of the action. (This is Gleason's lemma $[\mathbf{B r}, \mathrm{p}$. 36].) Let $A \equiv\{Y \in Y / G(Y, t)=0$, for some $t\}$. $A$ is invariant, $A \cap X=\varnothing$, so that one has a separating function $\lambda_{1}: Y \rightarrow[0,1]$, with $\lambda_{1}(A)=0, \lambda_{1}(X)=1$. Defining $\lambda(X)=(2 \pi)^{-1} \int_{0}^{2 \pi} \lambda_{1}\left(e^{i \phi} X\right) d \phi$, one gets an invariant separating function $(A$ and $X$ being invariants). In fact, by taking $\lambda(X)=\operatorname{dist}(X, A) /(\operatorname{dist}(X, B)+\operatorname{dist}(X, A))$ and since the action is an isometry, $\lambda$ is already invariant. The last step is to define $\tilde{G}(Y, t)=G(Y, \lambda(Y) t)$, which is easily seen to be a nonzero equivariant homotopy from $\tilde{F}_{0}$ to an equivariant extension of $F_{1}$.

For (2) it is enough to note that $(0, \ldots, 0, c)$ is $S^{1}$-inessential (it is important that the action has an invariant component; if not the only equivariant constant would be 0 ). Conversely, if $F$ is $S^{1}$-inessential with respect to $B$ (which is invariant under the sup-norm), then $H(X, t)=\tilde{F}((1-t) X)$ is an equivariant deformation of $F$ to a constant map, $\tilde{F}$ being the extension. Q.E.D.

Before applying the $S^{1}$-inessentiality to the global obstruction problem, it is worth it to note that this notion may be extended to any problem, on a general Banach space, with the action of any compact Lie group.

REMARK III.2.1. Inessentiality for general Lie groups. It is clear that the above definition and result can be extended to a more general situation. In fact let $G$ be a compact Lie group and $E$ be a Banach $G$-space. (I.e. $E$ is a representation of $G$ given by a continuous homomorphism $\rho: G \rightarrow \operatorname{GL}(E)$. Since $G$ is compact, one may assume that $\rho$ is an isometry on $E$ by renorming $E$ via $\|X\|^{\prime}=\int_{G}\|\rho(g)(X)\| d g, d g$ the normed Haar measure on $G$. For simplicity the action $\rho(g)(X)$ will be denoted 
by $g X$.) One will consider $G$-equivariant maps $(F(g X)=g F(X))$ of the form Identity-Compact and compact $G$-equivariant homotopies.

Definition III.2.1. Let $X$ be a closed, bounded, invariant subset of $E$ and $I-F$ be a $G$-equivariant map from $X$ into $E-\{0\}$ ( $F$ compact). $I-F$ is said to be $G$-inessential with respect to $Y, Y$ a closed, bounded, invariant set containing $X$, if $I-F$ extends to a $G$-equivariant map from $Y$ into $E-\{0\}$.

LEMMA III.2.1. (1) If $I-F_{0}$ and $I-F_{1}$ are equivariantly homotopic on $X$ and $I-F_{0}$ is $G$-inessential with respect to $Y$, then so is $I-F_{1}$.

(2) If the fixed point set of the action (which is a linear space) has positive dimension, write $E \equiv \tilde{E} \oplus \mathbf{R}\left(\mathbf{R}\right.$ a line in $\left.E^{G}\right)$, then $F$ is $G$-inessential with respect to any $Y$ (or with respect to a ball $B$ centered at the origin) if and only if $I-F \equiv$ $(I-\tilde{F}, f)$ is equivariantly deformable to $(\tilde{X}, c), c$ a nonzero constant in $\mathbf{R}$.

Proof. Tietze's extension theorem has to be replaced by Dugundji's and then one takes $G(X, t)=\int g^{-1} G(g X, t) d g$. For each fixed $X$, the orbit $g X$ is compact so that the integral is a well-defined equivariant extension. The only point is to check that if $G_{1}=I-K_{1}, K_{1}$ compact, then the resulting $G=I-K$ has $K$ compact. Thus it is enough to recall that $K$, on $Y$, is compact if an only if $K(X)=\sum_{0}^{\infty} K_{n}(X)$, where $K_{n}(X)$ belongs to a finite-dimensional space $E_{n}$ and $\left\|K_{n}(X)\right\| \leqslant 2^{-n}$ for all $X$ in $Y$ [Gr, Chapter II]. Let then $K_{1, n}$ be such a series for $K_{1}$ and define $K_{n}$ as its average on the group $G$. Then $\left\|K_{n}(X, t)\right\| \leqslant \operatorname{Sup}_{G}\left\|K_{1, n}(g X, t)\right\| \leqslant 2^{-n}$, since $Y$ is invariant and the action is an isometry. Clearly, $K_{n}(X, t)$ belongs to $G E_{n}$, is equivariant and the geometrical series converges to $K(X) . G E_{n}$ is a finite-dimensional $G$-subspace because the image under $G$ of the unit sphere is compact ( $G$ is compact and the action is continuous) and is the unit sphere in $G E_{n}$ (the action is an isometry). (One could also have used finite-dimensional approximation [Ni, Theorem 2.1.1; Ma, Lemma II.4].)

For $(2),(\tilde{X}, c)$ is clearly $G$-inessential and of the form $I$-compact. For the converse, note first that if $X-K(X)$ is the extension to $B$, then $X-$ $R^{-1}\|X\| K(X R /\|X\|)$ is a nonzero equivariant (the action is an isometry) extension for $\|X\| \geqslant R, R$ the radius of $B$. Now, since $X-K(X)$ is nonzero on $B$ and $K$ is compact, the above map is bounded away from zero on $B$ and there is a finitedimensional equivariant approximation $K_{N}$ of $K$, as above, which is equivariantly homotopic to $K$. If $K_{N}(B)$ is contained in $E_{N}$, a $G$-subspace, let $P_{N}$ be the projection on $E_{N}$ (one may assume that $\mathbf{R}$ is contained in $E_{N}$ and that $P_{N}$ is equivariant by averaging). Then

$$
X-K_{N}(X)=\left(I-P_{N}\right) X \oplus P_{N} X-K_{N}(X) .
$$

$K_{N}\left(P_{N} X+t\left(I-P_{N}\right) X\right)$ is a valid deformation. Then one may deform equivariantly $P_{N} X-K_{N}\left(P_{N} X\right)$ to $-K_{N}(0)$, a nonzero vector in $E_{N} \cap E^{G}$, which can be invariantly deformed to $(0, c)$. The deformation to $(\tilde{X}, c)$ is clear. Q.E.D. Note that if $E^{G}=\{0\}$, then $K(0)=0$ for any equivariant map, so that one cannot extend to $B$.

One may then use this on $W^{1,2}\left(S^{1}\right)$, defining the action on Fourier coefficients, or directly with the nonlinear action of translations along orbits. It is not difficult to check that averaging still works and is not necessary for autonomous functions. 


\section{III.3. The global obstruction.}

Since $\Omega_{N}$ is bounded, let $\bar{B}$ be a large ball $\left\{\left(Y, X_{0}, \mu, \nu\right) /\|Y\| \leqslant R_{1},\left\|X_{0}\right\| \leqslant R_{0}\right.$, $\left.\|\mu\| \leqslant R_{k}, c \leqslant \nu \leqslant C\right\}$ containing $\Omega_{N}$ ( $B$ is invariant from the sup-norm). For $E$ large enough, $F_{E}$ is nonzero on $\bar{B}$ (it is even possible to replace the first $(N+1)$ equations by any equivariant extension outside of $\Omega_{N}$ ). Thus $\left.F_{E}\right|_{\partial \Omega_{N}}$ is $S^{1}$-inessential with respect to $\bar{B}$ and $\left.F_{E}\right|_{\partial B}$ is equivariantly deformable to $(0,0,-E)$. Then for any $\varepsilon>0,\left.\left.F_{\varepsilon}\right|_{\partial \Omega_{N}} \cup(0,0,-E)\right|_{\partial B}$, which is equivariantly deformable on $\partial \Omega_{N} \cup \partial B$ to $\left.F_{E}\right|_{\partial \Omega_{v} \cup \partial B}$, is $S^{1}$-inessential on this pair of disjoint sets with respect to $\bar{B}$.

Fix $\varepsilon<\min \left(\varepsilon_{0}, \varepsilon_{1}\right)$. Let $F\left(Y, X_{0}, \mu, \nu\right) \equiv\left(F_{1}, \ldots, F_{N}, F_{0}, F_{r}\right)$, with $F_{j}$ in $\mathbf{C}^{M}$ for $j=1, \ldots, N, F_{0}$ in $\mathbf{R}^{M}, F_{r}$ in $\mathbf{R}$, be defined on $\bar{B}$ as (i) the extension of $\left.F_{\varepsilon}\right|_{\partial \Omega_{N}} \cup$ $\left.(0,0,-E)\right|_{\partial B}$ on $\bar{B}-\Omega_{N}$ and (ii) $F_{\varepsilon}$ on $\Omega_{N}$. Then $F\left(Y, X_{0}, \mu, \nu\right)$ is nonzero on $\bar{B}-\bigcup_{l} B(p)$, where $\bar{B}(p)=\left\{\|Y\| \leqslant \varepsilon_{1},\left\|X_{0}-X(p)\right\| \leqslant \varepsilon_{0},\left\|\mu-\mu_{p}\right\|+\left|T-T_{p}\right| \leqslant\right.$ $\left.\varepsilon_{k}\right\}$ is given in the construction of $\Omega$ (this comes from the choice of $\varepsilon$ : for any zero one has to be close to one of the bifurcation points). Let $\lambda=(\mu, \nu-(c+C) / 2), \lambda_{p}$ corresponding to $\left(\mu_{p}, \nu_{p}\right), R_{k}, \varepsilon_{k}$ will correspond to the different balls.

Consider the unit sphere $\left\{\left(\eta_{1}, \ldots, \eta_{N}\right), \eta_{j}\right.$ in $\mathbf{C}_{\tilde{B}}^{M}$ and $\left.\Sigma\left|\eta_{j}\right|^{2}=1\right\}$. Let the mapping from $S^{2 M N-1} \times \tilde{\bar{B}}$ into $\mathbf{C}^{M N} \times \mathbf{R}^{M} \times \mathbf{R}$, with $\tilde{\bar{B}}=\left\{\left(r, X_{0}, \lambda\right) / r \in \mathbf{R}, 0 \leqslant r \leqslant R_{1}\right.$, $\left.\left\|X_{0}\right\| \leqslant R_{0},\|\lambda\| \leqslant R_{k}\right\}$, be defined by

$$
\tilde{F}\left(\eta_{1}, \ldots, \eta_{N}, r, X_{0}, \lambda\right)=\left\{\begin{array}{l}
{\left[F_{j}\left(\tilde{r} \eta_{1}, \tilde{r} \eta_{2}^{2}, \ldots, \tilde{r} \eta_{N}^{N}, X_{0}, \lambda\right)\right]^{N ! / j}, \quad j=1, \ldots, N} \\
F_{0}\left(\tilde{r} \eta_{1}, \ldots, \tilde{r} \eta_{N}^{N}, X_{0}, \lambda\right) \\
F_{r}\left(\tilde{r} \eta_{1}, \ldots, \tilde{r} \eta_{N}^{N}, X_{0}, \lambda\right)
\end{array}\right.
$$

with $\tilde{r}=r\left(\max _{j}\left|\eta_{j}^{j}\right|\right)^{-1}$.

If $\tilde{\bar{B}}(p)=\left\{\left(r, X_{0}, \lambda\right) / 0 \leqslant r \leqslant \varepsilon_{1},\left\|X_{0}-X(p)\right\| \leqslant \varepsilon_{0},\|\lambda-\lambda(p)\| \leqslant \varepsilon_{k}\right\}$, then $\tilde{F} \neq 0$ on $S^{2 M N-1} \times\left(\bar{B}-\bigcup_{I} \tilde{B}(p)\right)$. In fact if $\left(\eta, r, X_{0}, \lambda\right)$ is a zero of $\tilde{F}$, then $\left(\tilde{r} \eta_{1}, \ldots, \tilde{r} \eta_{N}^{N}, X_{0}, \lambda\right)$ is a zero of $F$, with $\left\|\tilde{r} \eta_{1}, \ldots, \tilde{r} \eta_{N}^{N}\right\| \equiv \max _{j}\left|\tilde{r} \eta_{j}^{j}\right|=r$.

On $S^{2 M N-1}$ one has the standard action, while on the image in $\mathbf{C}^{M N} \times \mathbf{R}^{M} \times \mathbf{R}-$ $\{0\}$, one has the nonfree action $e^{i \phi}\left(Y, X_{0}, r\right) \equiv\left(e^{i N ! \phi} Y, X_{0}, r\right)$. After normalization, one gets a cross-section of the bundle

$$
S^{2 M N+M} \rightarrow K \times S^{2 M N-1} \times{ }_{S^{1}} S^{2 M N+M} \rightarrow K \times \mathbf{C} P^{M N-1},
$$

where $K=\overline{\bar{B}}-\cup_{I} \tilde{B}(p)$, extending the cross-section over $L \times \mathbf{C} P^{M N-1}$, with $L=$ $\partial(\tilde{B}-\bigcup, \tilde{B}(p))$. (The only difference with the previous case is that, having $2 M N+$ $M+1$ equations, the dimension of the fiber is different.) The eventual obstructions are all zero and belong to $H^{q+1}\left(K \times \mathbf{C} P^{M N-1}, L \times \mathbf{C} P^{M N-1} ; \Pi_{q}\left(S^{2 M N+M}\right)\right)$. Note that on $\partial \tilde{B}, \tilde{F}$ is $(0,0,-E)$ and, on $\partial \tilde{B}(p), \tilde{F}$ is

$$
\begin{aligned}
& {\left[\left(i j \nu I-L_{p}(\mu)(1-j /(N+1))\right) \tilde{r} \eta_{j}^{j}-g_{p, j}\left(\mu, Q_{N}\left(\tilde{r} \eta_{1}, \ldots, \tilde{r} \eta_{N}^{N}\right)\right)\right]^{N ! / j},} \\
& L_{p}(\mu)\left(X_{0}-X_{p}(\mu)\right)+g_{p .0}\left(\mu, Q_{N}\left(\tilde{r} \eta_{1}, \ldots\right)\right), \\
& d\left(\tilde{r} \eta_{1}, \ldots, \tilde{r} \eta_{N}^{N}, X_{0}, \mu, \nu ; S\right)-\varepsilon .
\end{aligned}
$$


On $\tilde{B}(p)$, the distance function is $\operatorname{Max}\left(r, \operatorname{Inf}_{\mu_{0}}\left(\left\|X_{0}-X_{p}\left(\mu_{0}\right)\right\|,\left\|\mu-\mu_{0}\right\|\right)\right)$, where $\left|\mu_{0}-\mu(p)\right| \leqslant \varepsilon_{k}$ ( $\nu$ does not enter).

III.4. Case of one bifurcation point on $\mathscr{C}$.

Since this case is easier to handle it will be treated first. Without loss of generality, one may take $\lambda_{p}=0, X(p)=0, R_{0}=R_{1}=R_{k}, \varepsilon_{0}=\varepsilon_{1}=\varepsilon_{k}$. For $\left(r, X_{0}, \lambda\right)$ in $\tilde{B}(0)$, define an extension of $\tilde{F}\left(\eta, r, X_{0}, \lambda\right)$ on $\partial \tilde{B}(0)$, by inversion with respect to its boundary; i.e.

$$
G\left(\eta, r, X_{0}, \lambda\right)=\tilde{F}\left(\eta, t r, t X_{0}, t \lambda\right)
$$

with $t\left(r, X_{0}, \lambda\right)=R_{0}\left\|r, X_{0}, \lambda\right\|^{-1}-\left(R_{0}-\varepsilon_{0}\right) \varepsilon_{0}^{-1}$. Then $\left\|t r, t X_{0}, t \lambda\right\|=R_{0}-$ $\left(R_{0}-\varepsilon_{0}\right)\left\|r, X_{0}, \lambda\right\| / \varepsilon_{0}$ (the value at the origin is $(0,0,-E)$, value of $\tilde{F}$ on $\left.\partial \tilde{B}\right)$. This means that $(\tilde{+})$ has an equivariant extension from $S^{2 M N-1} \times \partial \tilde{B}(0)$ to $S^{2 M N-1} \times$ $\tilde{B}(0)$ and a corresponding extension to the fiber bundle

$$
S^{2 M N+M} \rightarrow \tilde{B} \times S^{2 M N-1} \times{ }_{S_{1}} S^{2 M N+M} \rightarrow \tilde{B} \times \mathbf{C} P^{M N-1} .
$$

The eventual obstructions are in

$$
H^{k+M+1+2 p}\left(\tilde{B} \times \mathbf{C} P^{M N-1}, \partial \tilde{B} \times \mathbf{C} P^{M N-1} ; \Pi_{k+M+2 p}\left(S^{2 M N+M}\right)\right),
$$

$p=0, \ldots, 2 M N-2$, and they all vanish (the calculus of the cohomology groups is as before, with $k$ replaced by $k+M+1$, and the coefficient group has to be changed).

Recall that $\varepsilon_{0}, \varepsilon_{1}, \varepsilon_{k}$ have been chosen such that if $\|\lambda\|=\varepsilon_{k}$, the linear part of the equations for $j=1, \ldots, N$, dominates the nonlinear part (which is of the form $O\left(r\left(r+\left\|X_{0}\right\|\right)\right)$, since it vanishes for $r=0$ from the equivariance). For $\left\|X_{0}\right\|=\varepsilon_{0}$, then $\|X(\mu)\| \leqslant \varepsilon_{0} / 2$ (from the choice of $\varepsilon_{k}$ and continuity), so that $\left\|X_{0}-X(\mu)\right\| \geqslant$ $\varepsilon_{0} / 2$ and the linear part of the equation, for $j=0$, dominates the nonlinear part. For $r=\varepsilon_{1}$, the distance function is larger than $\varepsilon_{1}>\varepsilon$. For $r=0$, the only solution of the second equation is $X_{0}=X(\mu)$ (from the estimates on $g_{0}$ ) in which case the distance function is 0 . On $\partial \tilde{B}(0)$, one may then deform equivariantly the nonlinear parts to 0 . Then $L_{p}(\mu)$ can be deformed in $\operatorname{GL}\left(\mathbf{R}^{M}\right)$ to sign det $L_{p}(0) I$, the distance function to $r, \tilde{r}$ to $1,1-j /(N+1)$ to $1, X_{0}-X(\mu)$ to $X_{0}$, and the matrices $i j \nu I-L_{p}(\mu)$ to $I$ for all $j$ 's which are not of the form $m_{j}(p)$.

Now the pair $\left(\left(B(\lambda) \zeta^{j}\right)^{N ! / j}, \xi^{N !}\right)$ can be written as

$$
\left[\left(\begin{array}{ll}
B(\lambda) & \\
& I
\end{array}\right)\left(\begin{array}{l}
\zeta^{j} \\
\xi^{j}
\end{array}\right)\right]^{N ! / j} .
$$

Choose $N$ large enough so that one is dealing only with the stable classes of the matrices. If $k$ is odd or if $k>2 \tilde{d} \equiv 2 \max \operatorname{dim} \operatorname{ker} B_{j}(0)$, one may deform to $\left(\eta^{N !} \pm X_{0}, r-\varepsilon\right)$, which has an extension to $S^{2 M N-1} \times \tilde{B}(0)$ : the local invariant is 0 . However, if $k \leqslant 2 \tilde{d}$ and $k=2 s$, one has to look at the map

$$
\left(\eta, \lambda, X_{0}, r\right) \rightarrow\left(\tilde{\eta}^{N !},\left(C_{l}(\lambda) \xi_{l}^{m_{l}}\right)^{N ! / m_{l}}, \ldots,\left(C_{1}(\lambda) \xi_{1}^{m_{1}}\right)^{N ! / m_{1}},\right.
$$

$$
\text { sign det } \left.L_{p}(0) X_{0}, r-\varepsilon\right),
$$

with $\xi_{j} \in \mathbf{C}^{s}, \tilde{\eta} \in \mathbf{C}^{M N-l s}(s \leqslant M, l \leqslant N)$. 
The primary obstruction, for $p=M N-s$, is the degree of the mapping

$$
\begin{aligned}
\left(\tilde{\eta}, \xi_{l}, \ldots, \xi_{2}, \lambda, X_{0}, r\right) & \\
& \rightarrow\left(\tilde{\eta}^{N !},\left(C_{l}(\lambda) \xi_{l}^{m_{l}}\right)^{N ! / m_{l}},\left(C_{l-1}(\lambda) \xi_{l-1}^{m_{l-1}}\right)^{N ! / m_{l-1}}+t \xi_{l}^{N !}\right. \\
& \left.\quad \ldots,\left(C_{1}(\lambda)(1,0, \ldots, 0)^{T}\right)^{N ! / m_{1}}+t \xi_{2}^{N !}, \pm X_{0}, r-\varepsilon\right),
\end{aligned}
$$

where $t=\varepsilon_{0}-\left\|X_{0}, \lambda, r\right\|$, as it has been seen in §II.5.D. As before, this is the degree of $\left(\tilde{\eta}^{N !}, \xi_{1}^{N !}, \ldots, \xi_{2}^{N !}, \Pi C_{j}(\lambda)^{\left(N ! / m_{j}\right)^{s}}(1,0, \ldots, 0)^{T}\right.$, Sign det $\left.L(0) X_{0}, r-\varepsilon\right)$. By the product theorem, this degree is

$$
(N !)^{M N}(s-1) ! \text { Sign det } L_{p}(0) \sum_{j=1}^{l} n_{j}(k) / m_{j}^{s} .
$$

This gives the contradiction and the existence of the continuum.

REMARKS. III.4.1. $k=2$, this is the only obstruction, while for $k>2$, this is only the primary.

III.4.2. Existence of a local continuum. One may also prove the existence of a local continuum, even in the case of $k>2 \tilde{d}$ or $k$ odd, by remaining in a neighborhood of $(0,0,2 \pi / \beta)$ where the reduction is valid.

In the free case, $l=1$, one has to consider, as in Remark II.4.6, the class of $(\lambda, r) \rightarrow\left(\tilde{B}(\lambda) X_{1}, r-\varepsilon\right), \tilde{B}(0)=0, X_{1}$ fixed.

If the first suspension of $P^{*} B(\lambda)$ is nonzero, one gets a local continuum (if the suspension is zero it would be interesting to know if no continuum exists).

For the nonfree case, one may use the reduction given in Remark II.5.2. The only nontrivial case is for $k$ odd, $k \leqslant 2 \tilde{d}+1$, giving a primary obstruction in $\Pi_{n}\left(S^{n-1}\right)$ with $n \geqslant 4$ (since $l>1$, one has at least a 2-complex dimensional problem). By adding $r-\varepsilon$ one obtains the suspension in the stable range.

If the local invariant, given in Theorem II.5.1, is nonzero, one has a local continuum.

III.5. General case.

In this section one will relate the local to the global obstructions, which are 0 in $H^{q+1}\left(\cdot, \cdot ; \Pi_{q}\left(S^{2 M N+M}\right)\right)$. The cell-complexes are much more complicated, but the only local obstruction which is of value is the primary obstruction, for $q=2 M N+M$ and $k=2 s$. In this case, the obstruction is given by $\delta_{0} \kappa^{2 M N+M}(\tilde{F})$, where $\delta_{0}$ is the coboundary operator

$$
H^{2 M N+M}\left(L \times \mathbf{C} P^{M N-1}\right) \rightarrow H^{2 M N+M+1}\left(K \times \mathbf{C} P^{M N-1}, L \times \mathbf{C} P^{M N-1}\right)
$$

and $\kappa(\tilde{F})$ is the characteristic element of $\tilde{F}$.

Locally one has to replace $(K, L)$ by $(\tilde{B}(p), \partial \tilde{B}(p))$. Then one may follow the argument given in [14, p. 159] for cohomology instead of cohomotopy:

Consider first the exact sequence for the pair $\left(B \times C P^{M N-1}, \partial B \times C P^{M N-1}\right)$. Since $B$ is contractible and the cohomology for $C P^{M N-1}$ is 0 in dimension larger than $2 M N-2$, the coboundary operators $\delta(p)$ (for $\tilde{B}(p)$ ) and $\delta$ (for $\tilde{B}$ ) are isomorphisms in dimension $2 M N+M, H^{2 M N\lrcorner M}\left(\partial B \times C P^{M N-1}\right)=Z$, the unique relative cell being of the form $B^{M+k+1} \times B^{2 M N-2 s}$. 
Secondly, by excision of the small balls $\tilde{B}(p)$, one has

$$
\begin{aligned}
H^{q}\left(K \times \mathbf{C} P^{M N-1}, \cup_{I} \partial \tilde{B}(p) \times\right. & \left.\mathbf{C} P^{M N-1}\right) \\
& \cong H^{q}\left(\tilde{\bar{B}} \times \mathbf{C} P^{M N-1}, \cup_{I} \tilde{\bar{B}}(p) \times \mathbf{C} P^{M N-1}\right) \cong 0,
\end{aligned}
$$

for $q \geqslant 2 M N$ (using the cohomology sequence for this last pair and the facts that $\tilde{B}$, $\tilde{B}(p)$ are contractible and the dimension of $\left.C P^{M N-1}\right)$. Thus the mapping $j^{*}$, induced by inclusion

$$
H^{2 M N+M}\left(K \times \mathbf{C} P^{M N-1}\right) \stackrel{j^{*}}{\rightarrow} H^{2 M N+M}\left(\bigcup_{I} \partial \tilde{B}(p) \times \mathbf{C} P^{M N-1}\right)
$$

is an isomorphism.

Furthermore in the diagram

$$
\begin{array}{ccc}
H^{2 M N+M}\left(K \times \mathbf{C} P^{M N-1}\right) & \stackrel{k^{*}}{\rightarrow} & H^{2 M N+M}\left(\partial \tilde{B} \times \mathbf{C} P^{M N-1}\right) \\
\uparrow i_{p}^{*} & j_{p}^{*} \nearrow & \\
H^{2 M N+N}\left((\tilde{\bar{B}}-\tilde{B}(p)) \times C P^{M N-1}\right) & \stackrel{j_{p}^{*}}{\rightarrow} & H^{2 M N+M}\left(\partial \tilde{B}(p) \times \mathbf{C} P^{M N-1}\right)
\end{array}
$$

where all maps are induced by inclusions, $j_{p}^{*}, \tilde{j}_{p}^{*}$ are isomorphisms by radial retraction from $\tilde{B}-\tilde{B}(p)$ onto $\partial \tilde{B}$ (or $\partial \tilde{B}(p)$ ). Hence, $k^{*}$ is onto (or $k_{p}^{*}$ is onto, where $\tilde{B}$ is replaced by $\tilde{B}(p))$. Note that $j^{*}=\bigoplus_{I} k_{p}^{*}$.

Consider now the commutative diagram:

$$
\begin{aligned}
& \oplus_{I} H^{2 M N+M}\left(\partial \tilde{B}(p) \times \mathbf{C} P^{M N-1}\right) \\
& \lambda j^{*} \quad \uparrow i_{1}^{*} \\
& 0 \rightarrow H^{2 M N+M}\left(K \times \mathbf{C} P^{M N-1}\right) \stackrel{i_{0}^{*}}{\rightarrow} \quad \begin{array}{l}
H^{2 M N+M}\left(L \times \mathbf{C} P^{M N-1}\right) \\
\stackrel{\delta_{0}}{\rightarrow} H^{2 M N+M+1}\left(K \times \mathbf{C} P^{M N-1}, L \times \mathbf{C} P^{M N-1}\right) \rightarrow 0
\end{array} \\
& \begin{array}{cc}
\searrow k^{*} & \downarrow i_{2}^{*} \\
& H^{2 M N+M}\left(\partial \tilde{B} \times C P^{M N-1}\right)
\end{array}
\end{aligned}
$$

(That $i_{0}^{*}$ is one-to-one and $\delta_{0}$ is onto follows from the fact that $j^{*}$, and hence $i_{0}^{*}$, is one-to-one at the levels $2 M N+M$ and $2 M N+M+1$.) Since $L=\partial \tilde{B} \cup_{I} \partial \tilde{B}(p)$, $i^{*} \equiv i_{1}^{*} \oplus i_{2}^{*}$ is an isomorphism and, since $\kappa(F)=F^{*}\left(\kappa\left(S^{2 M N+M}\right)\right)$, one has $\kappa\left(\left.\tilde{F}\right|_{\partial \tilde{B}}\right)=i_{2}^{*} \kappa(\tilde{F}), \oplus \kappa(\tilde{F}(p))=i_{1}^{*} \kappa(\tilde{F})$. If $\alpha=i_{0}^{*} \beta$, then $i_{1}^{*} \alpha=j^{*} \beta, \beta=j^{*-1} i_{1}^{*} \alpha=$ $j^{*-1} i_{1}^{*} i_{0}^{*} \beta$, so that $\operatorname{Im} i_{0}^{*}=\operatorname{ker}\left(I-i_{0}^{*} j^{*-1} i_{1}^{*}\right)$. Also $i^{*}\left(I-i_{0}^{*} j^{*-1} i_{1}^{*}\right) \kappa(\tilde{F})=0 \oplus$ $\kappa\left(\left.\tilde{F}\right|_{\partial \tilde{B}}\right)-k^{*} j^{*-1} \oplus_{I} \kappa(\tilde{F}(p))$, so that $\delta_{0}(\kappa(\tilde{F}))=0$ if and only if $\kappa\left(\left.\tilde{F}\right|_{\partial \tilde{B}}\right)=$ $k^{*} j^{*-1} \oplus_{I} \kappa(\tilde{F}(p))$.

Here $\left.\tilde{F}\right|_{\partial \tilde{B}} ^{I}=(0,0,-E)$ is trivial and the global obstruction is 0 , i.e. $\delta_{0}(\kappa(\tilde{F}))=0$. Furthermore,

$$
k^{*} j^{*-1} \bigoplus_{I}=\sum_{I} j_{p}^{*} \tilde{j}_{p}^{*-1},
$$

from the first diagram. Using the inclusions of $(\tilde{B}, \partial \tilde{B})$ and $(\tilde{B}(p), \partial \tilde{B}(p))$ in $(\tilde{B}, \tilde{B}-\tilde{B}(p))$ and the commutativity of the sequences for these pairs, one has $\delta j_{p}^{*} \tilde{j}_{p}^{*-1}=j_{p}^{*} \tilde{\delta} \tilde{j}_{p}^{*-1}=j_{p}^{*} \tilde{j}_{p}^{*-1} \delta_{p}\left(\delta, \delta_{p}, \tilde{\delta}\right.$ are the operators for each of the above pairs $)$. One thus obtains

$$
\sum_{I} j_{p}^{*} \tilde{j}_{p}^{*-1} C(\tilde{F}(p))=0
$$


But, from the retractions, $j_{p}^{*} \tilde{j}_{p}^{*-1}$ sends generators in generators, so that the sum of the local indices (which have been already computed in the last section) must be 0 , i.e.

$$
\sum_{I} \operatorname{sign} \operatorname{det} L_{p}\left(\mu_{p}\right) \sum_{j=1}^{l_{p}} \frac{n_{j}(k, p)}{\left(m_{j}(p)\right)^{k / 2}}=0 .
$$

This ends the proof of the Main Theorem.

IV. Concluding remarks.

IV.1. In [MY], a summation formula for a generic case $\left(k=2, m_{j}(p)=1, l_{p}=1\right)$ is announced without proof. After this work was completed the author learned of an extension of the ideas in [MY] for the partial differential equation case given in [Fi]. Extensions of the method given in the present paper to the continuation problem or to the infinite-dimensional case do not seem to be very difficult and will be the subject of a subsequent paper.

IV.2. After completing this paper, the author was informed by Professors Berestycki and Lasry of a result of S. Y. Husseini on the homotopy group of equivariant maps between spheres [Hus].

Husseini considers the map

$$
\left(X_{0}, B_{1}(\lambda) X_{1}, \ldots, B_{l}(\lambda) X_{l}\right)
$$

defined on $\left|X_{0}\right|^{2}+|Y|^{2}=1$, with $Y^{T}=\left(X_{1}, \ldots, X_{l}\right), k=2 s \leqslant 2 \tilde{d}$. Then, he looks for equivariant extensions of (15) from the set $\{|\lambda|=1\}$ to the set $\{|\lambda| \leqslant 1\}$, in such a way that the invariant component of this extension has the property that $f_{0}\left(X_{0}, \lambda, 0\right)=X_{0}$. His main result is that if such an extension exists, then the degree in formula (14) is 0 .

Although some of the ideas are similar, several comments are in order.

(a) After taking the product with the universal classifying space, Husseini reduces the problem to an equivariant obstruction problem and computes the equivariant cohomology of pairs of spheres. The machinery from Algebraic Topology is much heavier than the techniques used in this paper.

(b) The main difference in spirit between the two papers is that Husseini has an approach which is more natural from the topological point of view, whereas the present approach is closer to analysis. For instance, since one is looking for nontrivial solutions $(Y \neq 0)$, it is natural to consider the invariant component $X_{0}$ as a set of new parameters. Hence one studies (15) on the set $\{|Y|=1\} \times\left\{\left|X_{0}\right|^{2}+|\lambda|^{2}\right.$ $=1\}$ and equivariant extensions to the set $\{|Y|=1\} \times\left\{\left|X_{0}\right|^{2}+|\lambda|^{2} \leqslant 1\right\}$.

(c) In $\S \S I I .5$ and III.3, it has been proved that if the expression

$$
\left(X_{0}, r B_{1}(\lambda) \eta_{1}, \ldots, r B_{l}(\lambda) \eta_{l}, r-1\right),
$$

defined on the set $\{|\eta|=1\} \times \partial\left\{\left|X_{0}\right|^{2}+|\lambda|^{2} \leqslant 1,0 \leqslant r \leqslant 2\right\}$, has an equivariant extension to $\{|\eta|=1\} \times\left\{\left|X_{0}\right|^{2}+|\lambda|^{2} \leqslant 1,0 \leqslant r \leqslant 2\right\}$, then the degree of (14) is 0 .

It is not difficult to show that (16) extends, as above, if and only if

$$
\left(X_{0}, B_{1}(\lambda) X_{1}, \ldots, B_{l}(\lambda) X_{l},|Y|-1\right)
$$


extends equivariantly from $\left\{\left|X_{0}\right|^{2}+|Y|^{2}+|\lambda|^{2}=2\right\}$ to $\left\{\left|X_{0}\right|^{2}+|Y|^{2}+|\lambda|^{2} \leqslant 2\right\}$, provided the invariant component has the property that $f_{0}\left(X_{0}, \lambda, 0\right)=X_{0}$.

Consider finally

$$
\left(X_{0}, B_{1}(\lambda) X_{1}, \ldots, B_{l}(\lambda) X_{l},\left|X_{0}\right|^{2}+|Y|^{2}-1\right) .
$$

It is easy to show that (17) and (18) are equivariantly homotopic on the above 'sphere, provided the homotopy preserves the condition $f_{0}\left(X_{0}, \lambda, 0\right)=X_{0}((18)$ corresponds to the $J$-homomorphism image of (15) under Husseini's approach, while (17) corresponds to the image under the present approach).

This imples that, if (15) extends in Husseini's context, then so does (18), hence (17), and also (16). The degree of (14) is 0 . Thus Husseini's result is a consequence of this paper.

(d) Husseini does not consider the global obstruction nor the unstable case.

(e) One may improve the Berestycki-Lasry application on the existence of $2 \pi$ periodic solutions to the system $\dot{X}=\nu \phi(X), X$ in $\mathbf{R}^{2 m}, \phi(X) \cdot X=0, \phi(0)=0$, $\phi(X) \cdot \sigma X>0$, where $\sigma$ is an antisymmetric regular matrix, $|\phi(X)-\sigma X|<\alpha|X|(\alpha$ depending on the spectrum of $\sigma$ ), by proving the existence of continua going to infinity.

IV.3. If $L(0)$ is singular, one may have bifurcation of stationary solutions. However, it is possible to put conditions on the nonlinearity to guarantee the existence of periodic solutions. In this case the bifurcation equation has an invariant component. The details will be given in a later paper.

IV.4. Nirenberg, in [Ni2], uses a trick, similar to the one needed to study the nonfree case, to compute the degree of an $S^{1}$-equivariant mapping. Using an argument of dimension, it is not difficult to prove that in fact this degree is the product of the degree of the invariant component by the formula given in [Ni2]. (There the invariant component has the property that $f\left(X_{0}, 0\right)=X_{0}$, with degree 1.)

IV.5. Finally, it is clear that the method of this work can be extended to other group actions, either finite or infinite, defining an equivalent of Fuller's degree.

\section{REFERENCES}

[A] J. C. Alexander, Bifurcation of zeros of parametrized functions, J. Functional Anal. 29 (1978), 37-53.

[AY] J. C. Alexander and J. A. Yorke, Global bifurcation of periodic orbits, Amer. J. Math. 100 (1978), 263-292.

[At] M. F. Atiyah, Algebraic topologv and elliptic operators, Comm. Pure Appl. Math. 20 (1967), 235-249.

[Bo] A. Borel, Topology of Lie groups and characteristic classes, Bull. Amer. Math. Soc. 61 (1955), 397-432.

[Br] G. E. Bredon, Introduction to compact transformation groups, Academic Press, New York, 1972.

[CMY] S. N. Chow, J. Mallet-Paret and J. A. Yorke, Global Hopf bifurcation from a multiple eigenvalue, Nonlinear Anal. 2 (1978), 755-763.

[FR] F. R. Fadell and P. H. Rabinowitz, Generalized cohomological index theories for Lie group actions with an application to bifurcation questions for Hamiltonian systems, Invent. Math. 45 (1978), 134-174.

[Fi] B. Fiedler, Stabilitätswchsel und globale Hopf-Verzweingung, Dissertation, Heidelberg, August 1982.

[Fu] F. B. Fuller, An index of fixed point type for periodic orbits, Amer. J. Math. 89 (1967), 133-148.

[Gr] A. Granas, The theory of compact vector fields and some of its applications to topology of functional spaces. I, Rozprawy Mat. 30 (1962).

[Hu] S. T. Hu, Homotopy theory, Academic Press, New York, 1959. 
[Hus] S. Y. Husseini, The equivariant J-homomorphism for arbitrary $S^{1}$-action, University of Wisconsin, Preprint, 1982.

[I1] J. Ize, Bifurcation theory for Fredholm operators, Mem. Amer. Math. Soc. 7, No. 174, (1976).

[I2] (1979), 1299-1387. , Periodic solutions of nonlinear parabolic equations, Comm. Partial Differential Equations 4

[I3] , Le problème de bifurcation de Hopf, Sem. Brezis-Lions, 1975 (A paraitre).

[14] Introduction to bifurcation theory, in Proceedings of the 1st. Latin-American School of Differential Equations, Lecture Notes in Math., vol. 957, Springer-Verlag, Berlin and New York, 1982, pp. 145-203.

[IMPV] J. Ize, I. Massabó, J. Pejsachowicz and A. Vignoli, Structure and dimension of global branches of solutions to multiparameter nonlinear equations, Preprint in Comunicaciones Técnicas No. 350 (1983).

[Ko] C. Kosnioswski, Equivariant cohomology and stable cohomotopy, Math. Ann. 210 (1974), 83-104.

[Ku] K. Kuratowski, Topology, Vol. 2, Academic Press, New York, 1968.

[MY] J. Mallet-Paret and J. A. Yorke, Snakes: oriented families of periodic orbits, their sources, sinks and continuation, J. Differential Equations 43 (1982), 419-450.

[Ma] W. Marzantowicz, On the nonlinear elliptic equations with symmetry, J. Math. Anal. Appl. 81 (1981), 156-181.

[Mt] T. Matsuoka, Equivariant function spaces and bifurcation points, J. Math. Soc. Japan. 35 (1983), 43-52.

[Ni] L. Nirenberg, Topics in nonlinear functional analysis, Courant Institute Lecture Notes, 1974.

[Ni2] , Comments on nonlinear problems, Le Matimatiche 36 (1981), 109-119.

[Pa] R. S. Palais, The classification of G-spaces, Mem. Amer. Math. Soc. No. 36, (1960).

[Sp] E. Spanier, Algebraic topology, McGraw-Hill, New York, 1966.

[St] N. Steenrod, Topology of fiber bundles, Princeton Univ. Press, Princeton, N.J., 1965.

[To] H. Toda, Composition methods in homotopy groups of spheres, Ann. of Math. Studies, No. 49. Princeton Univ. Press, Princeton, N.J., 1962.

IIMAS - UNAM, A.P. 20 - 726, MEXICo 20, D.F. 